\title{
Macrophages-aPKC,-CCL5 Feedback Loop Modulates the Progression and Chemoresistance in Cholangiocarcinoma
}

Tao Yang ${ }^{1,2+}$, Zhengdong Deng ${ }^{1 \dagger}$, Lei Xu ${ }^{1}$, Xiangyu Li ${ }^{1}$, Tan Yang ${ }^{3}$, Yawei Qian ${ }^{4}$, Yun Lư ${ }^{1}$ Li Tian ${ }^{1}$, Wei Yao ${ }^{1,5^{*}}$ and Jianming Wang ${ }^{1,6^{*}}$

\begin{abstract}
Background: Recent data indicated that macrophages may mutually interact with cancer cells to promote tumor progression and chemoresistance, but the interaction in cholangiocarcinoma (CCA) is obscure.

Methods: 10x Genomics single-cell sequencing technology was used to identified the role of macrophages in CCA. Then, we measured the expression and prognostic role of macrophage markers and $\mathrm{APKC}_{1}$ in 70 human CCA tissues. Moreover, we constructed monocyte-derived macrophages (MDMs) generated from peripheral blood monocytes (PBMCs) and polarized them into M1/M2 macrophages. A co-culture assay of the human CCA cell lines (TFK-1, EGI-1) and differentiated PBMCs-macrophages was established, and functional studies in vitro and in vivo was performed to explore the interaction between cancer cells and M2 macrophages. Furthermore, we established the cationic liposome-mediated co-delivery of gemcitabine and $\mathrm{PPKC}_{1}-\mathrm{siRNA}$ and detect the antitumor effects in CCA.
\end{abstract}

Results: M2 macrophage showed tumor-promoting properties in CCA. High levels of aPKC $C_{1}$ expression and M2 macrophage infiltration were associated with metastasis and poor prognosis in CCA patients. Moreover, CCA patients with low M2 macrophages infiltration or low $\mathrm{PPKC}_{1}$ expression benefited from postoperative gemcitabine-based chemotherapy. Further studies showed that M2 macrophages-derived TGF $\beta 1$ induced epithelial-mesenchymal transition (EMT) and gemcitabine resistance in CCA cells through aPKC--mediated NF-KB signaling pathway. Reciprocally, CCL5 was secreted more by CCA cells undergoing $\mathrm{APKC}_{1}$-induced EMT and consequently modulated macrophage recruitment and polarization. Furthermore, the cationic liposome-mediated co-delivery of GEM and aPKC,-siRNA significantly inhibited macrophages infiltration and CCA progression.

Conclusion: our study demonstrates the role of Macrophages-aPKC 1 -CCL5 Feedback Loop in CCA, and proposes a novel therapeutic strategy of aPKC,-siRNA and GEM co-delivered by liposomes for CCA.

Keywords: Cholangiocarcinoma, Positive Feedback Loop, Macrophages, $\mathrm{aPKC}_{{ }^{\prime}}$ Chemoresistance

*Correspondence: yw13557@163.com; wjm18jgm@aliyun.com

${ }^{\dagger}$ Tao Yang and Zhengdong Deng contributed equally to this work. ${ }^{1}$ Department of Biliary and Pancreatic Surgery/Cancer Research Center Affiliated Tongji Hospital, Tongji Medical College, Huazhong University of Science and Technology, Wuhan 430030, Hubei, China

Full list of author information is available at the end of the article

\section{Background}

Cholangiocarcinoma (CCA) is one of the most highly malignant and lethal cancers with limited overall survival and is notorious for its rapid progression, early metastasis and therapeutic resistance. Although radical resection is the most effective approach for prolonging long-term survival, more than two-thirds of patients lack operative opportunities for locally advanced or distant metastatic 
tumors. Moreover, even with aggressive surgical treatment, the 5-year survival rates of CCA patients remain unsatisfactory $[1,2]$. Gemcitabine (GEM) based chemotherapy is the first-line of care treatment for CCA, but it does not significantly improve the overall survival of CCA patients [3, 4]. Therefore, there is a desperate need to identify the mechanism of chemoresistance and develop improved therapeutic strategies for CCA patients.

The tumor microenvironment (TME) plays a significant role in promoting cancer progression, invasion, metastasis and chemoresistance [5]. Macrophages in the TME, namely tumor-associated macrophages (TAMs), exhibit different phenotypes and functional features owing to pathogen or cytokine stimulation. TAMs often display an alternatively activated (M2) phenotype and enhance tumor malignancy in the majority of cases [6], and cancer cells can actively modulate the recruitment and activation of macrophages to enhance tumor growth and metastasis $[7,8]$. CCA is characteristically marked by a highly desmoplastic and hypovascularized microenvironment [9]. The prognostic and clinical significance of TAMs has previously been reported in CCA, and TAMs are correlated with poor prognosis and dismal survival outcomes [10]. Furthermore, Kitano et al. [11] identified a risk signature, derived from the integration of intratumoral neutrophils, macrophages, $\mathrm{CD} 8^{+} \mathrm{T}$ cells and Tregs, related to GEM chemoresistance in CCA patients. Noteworthy, accumulated evidence has shown that cancer cells and stromal cells interact in tumor microenvironment to regulate tumor growth and progression and offer a potential treatment strategy for cancer patients [12]. Ljichi et al. [13] reported that targeting tumor-stromal interactions could reduce chemoresistance and improve survival in a mouse model of pancreatic ductal adenocarcinoma. However, the role and mechanism of the crosstalk between TAMs and cancer cells in CCA are still unclear.

It has been demonstrated that epithelial-mesenchymal transition (EMT), a process whereby differentiated epithelial cells lose their polarity and acquire a mesenchymal phenotype, is predominantly associated with chemoresistance, cancer stem cells and the immune microenvironment [14]. We previously demonstrated that atypical protein kinase $\mathrm{C}$ iota $(\mathrm{aPKCl})$ promotes $\mathrm{EMT}$ and induces immunosuppression in $\mathrm{CCA}$, suggesting that aPKCı may play a pivotal role in the interaction between cancer cells and the TME [15]. Herein, we provide the first report of a positive feedback loop between macrophages and cancer cells that promotes CCA progression and chemoresistance. Our results indicate that M2 macrophages secrete TGF $\beta 1$ to induce cancer cell EMT and chemoresistance through the $\mathrm{aPKC}_{1}-\mathrm{NF}-\mathrm{kB}$ signaling pathway in CCA. Reciprocally, CCL5 levels are significantly increased in the supernatant of CCA cells that undergo ${ }_{a P K C}$-induced EMT and consequently modulate the recruitment and activation of macrophages.

Combination therapy with siRNAs and chemotherapeutic drugs has been considered as an alternative option to enhance the anticancer efficiency $[16,17]$. Other groups, as well as us, have investigated whether the co-delivery of chemotherapeutic drugs and siRNAs by liposome- or nanoparticle-based systems could be a promising strategy for overcoming chemoresistance in breast cancer and pancreatic cancer [16, 18]. Given the crucial roles of $\mathrm{aPKC}_{1}$, we prepared cationic liposomes to co-deliver GEM and $\mathrm{aPKC}_{1}$-siRNA to improve the chemotherapeutic responses and treatment efficacy of CCA.

In the study, we describe the interaction between macrophages and CCA cells, and provide a novel combination therapy strategy with aPKC ${ }_{1}$-siRNA and GEM using a liposome-based drug delivery system for CCA patients.

\section{Results \\ The clinical significance of macrophages infiltration and $\mathrm{APKC}$ in human CCA}

To better understand the role of macrophages in the cholangiocarcinoma tumor environment (TME), we employed the 10x Genomics single-cell sequencing technology to reveal the phenotype and function of myeloid immune cells in human cholangiocarcinoma. As shown in Fig. 1A, the myeloid immune cells in CCA tumor and paratumor tissues are mainly divided into 4 cell subtypes after dimensionality reduction cluster analysis: M1 macrophage subtype, M2 macrophage subtype, a classical dendritic cell subtype and a plasmacytoid dendritic cell subtype. We found that the proportion of M1 macrophages in tumor tissue was lower than that in paratumor tissue (42.18\% vs $70.09 \%)$, whereas the proportion of M2 macrophages was higher in tumor tissue $(25.09 \%$ vs $2.78 \%$ ). Despite a greater proportion of M1 macrophages in the TME, M2 macrophages expressed higher gene

\footnotetext{
(See figure on next page.)

Fig. 1 The clinical significance of macrophages infiltration and aPKCI in human CCA. A. Reclassification of Myeloid Immune Cell in Cholangiocarcinoma based on Single Cell RNA Sequencing. B. The expression of TAMs markers and some cancer-promoting genes in each Macrophages subtypes. C. IHC was used to examine the levels of aPKC $(1: 200)$ and macrophage markers such as CD68 (1:150), CD80 (1:500), and CD206 (1:400) in 70 paired CCA and paratumor tissues. Representative images are shown. Scale bar, $20 \mu \mathrm{m}$. D. Quantification of aPKC, and macrophage markers expression level in paired CCA and paratumor tissues. E. Kaplan-Meier analysis indicating the correlation between the prognosis of CCA patients and the expression of $\mathrm{aPKC}_{v^{\prime}} \mathrm{CD} 68, \mathrm{CD} 80$ and $\mathrm{CD} 206$
} 
A

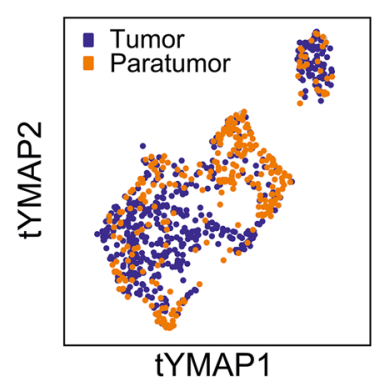

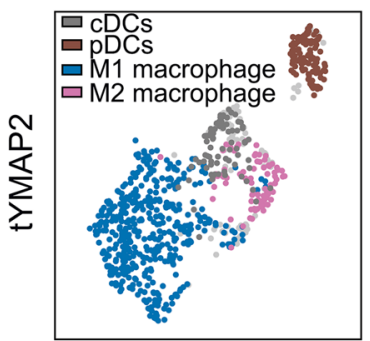

tYMAP1
B

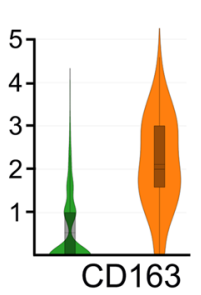

- M1 macrophage
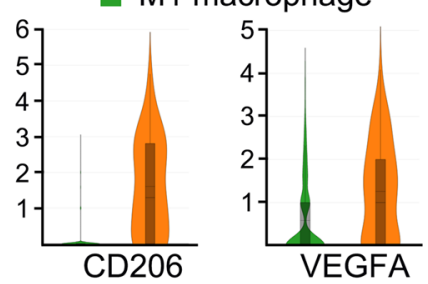
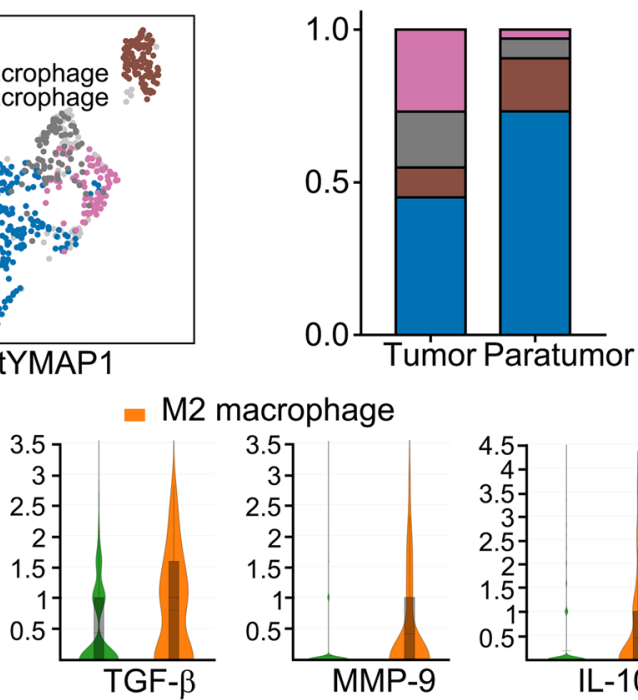

M2 macrophage

C

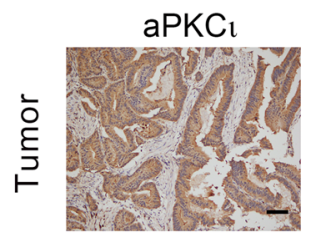

CD68

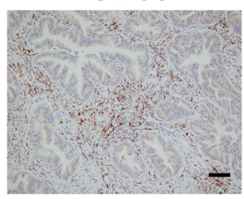

CD80
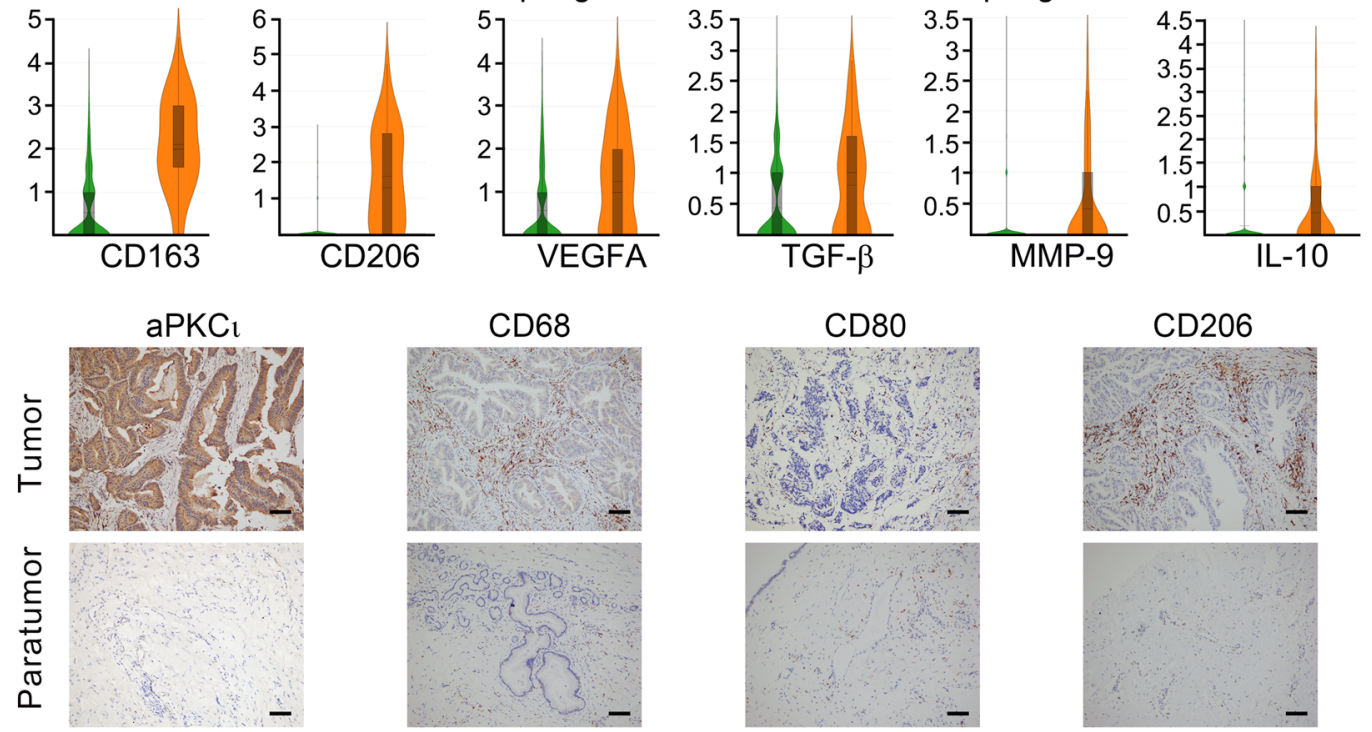

CD206

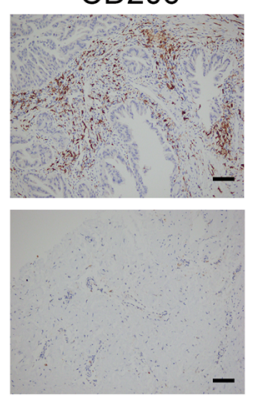

D
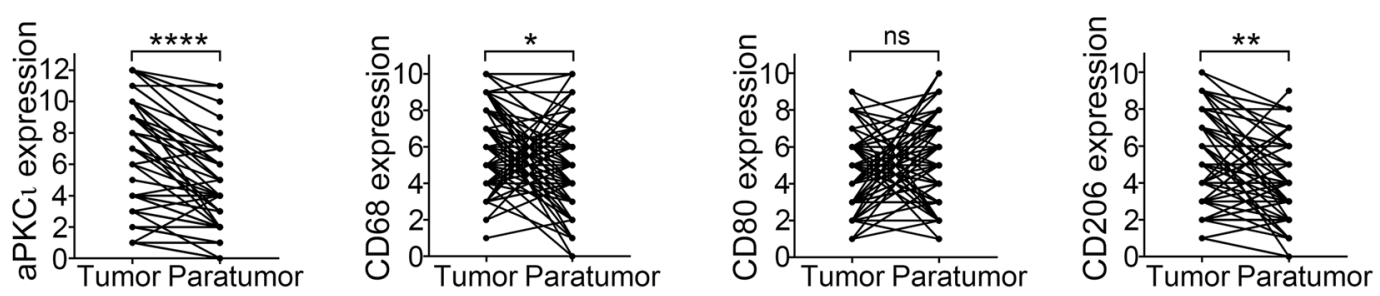

E
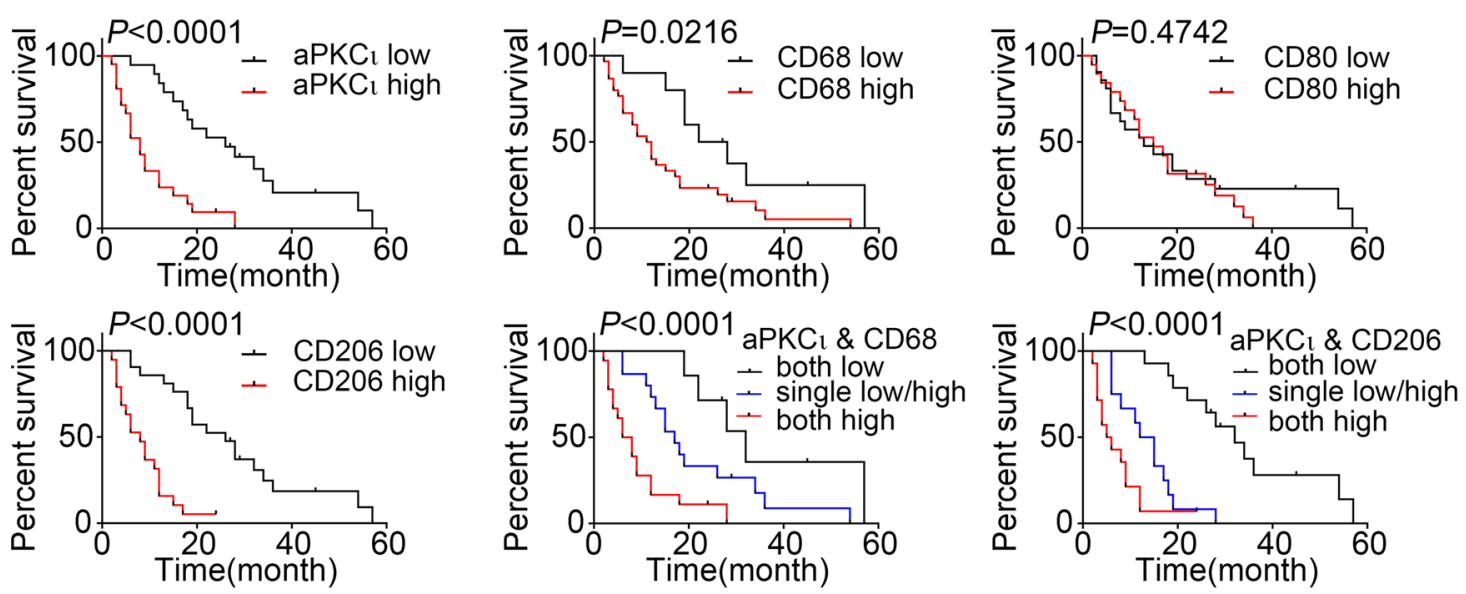

Fig. 1 (See legend on previous page.) 
level of CD163, MRC1 (CD206), TGF- $\beta$, IL10, VEGFA, and MMP9, which exhibited stronger tumor-promoting ability (Fig. 1B).

To investigate the clinical significance of macrophage infiltration and $\mathrm{aPKC}_{1}$ expression in human CCA, we examined aPKCl and TAMs markers (macrophage: CD68; M1 macrophage: CD80; M2 macrophage: CD206) expressed in human CCA by immunohistochemistry (IHC), Western blotting (WB), and quantitative real-time polymerase chain reaction (qRT-PCR). The IHC analysis showed that staining of aPKCl, CD68, and CD206 was enriched in CCA tissues than pair-matched paratumor tissues (Fig. 1C-D). Consistent results were verified by WB and qRT-PCR (Supplementary Fig. 1A-B). Then, we analyzed the association between $\mathrm{aPKC}_{1}$ and TAMs markers in CCA specimens. Pearson correlation analyses of the above IHC results confirmed that expression of aPKC $\mathrm{A}_{1}$ was positively correlated with CD68 $(\mathrm{r}=0.4128$, $P=0.0004)$ and CD206 $(r=0.5489, P<0.0001)$. However, there was no significant correlation between $\mathrm{aPKC}_{1}$ and CD80 ( $\mathrm{r}=0.0540, P=0.6569)$ (Supplementary Fig. 1C).

We further explored the correlation of aPKCi expression and macrophage infiltration with the clinicopathological characteristics and prognosis in patients with CCA. Notably, overexpression of $\mathrm{aPKC}_{1}$ and CD206 was remarkably associated with lymph node metastasis $\left(\chi^{2}=6.005,4.086 ; P=0.014, P=0.043\right.$, respectively), tumor-node-metastasis (TNM) stage III-IV $\left(\chi^{2}=6.740\right.$, 12.899; $P=0.009, P<0.001)$, and moderate/poor differentiation $\left(X^{2}=3.994,4.073 ; P=0.046, P=0.044\right)$. High level of CD68 was just related to lymph node metastasis $\left(\chi^{2}=4.076 ; \quad P=0.044\right) \quad$ and $\quad$ tumor-node-metastasis (TNM) stage III-IV $\left(X^{2}=5.871 ; P=0.015\right)$, while CD80 was not associated with clinicopathological characteristics (Table 1). Moreover, a Kaplan-Meier analysis revealed that patients with high level of aPKC, CD68 or CD206 rather than CD80 displayed worse overall survival (OS). Prognosis was also statistically associated with the co-expression of aPKCı and CD68/CD206 (Fig. 1E). Multivariate Cox regression analyses indicated that aPKCt and CD206 were independent prognostic factors for OS in CCA patients (Table 2). These data imply that aPKCı and $\mathrm{CD}_{206^{+}}$macrophage (M2 macrophage), but not $\mathrm{CD}^{+} 0^{+}$macrophage (M1 macrophage), may contribute to the progression and dismal prognosis of CCA.

\section{M2 macrophages induce aPKCI-mediated CCA cell chemoresistance to GEM}

To study the contribution of $\mathrm{M} 2$ macrophages and aPKCt to chemoresistance, we investigated the efficacy of postoperative GEM-based chemotherapy in CCA patients. As shown in Supplementary Fig. 2A, chemotherapy did not provide apparent survival benefit in CCA patients. However, we found patients with high aPKCı expression exhibited no response to postoperative chemotherapy, while patients with low aPKCı expression responded well (Fig. 2A). Consistently, patients with low $\mathrm{CD}^{206^{+}}$macrophage infiltration displayed a favorable outcome after postoperative chemotherapy, whereas no apparent benefit was found in patients with high $\mathrm{CD}_{20}{ }^{+}$macrophage infiltration (Fig. 2A). These findings indicate that aPKCl

Table 1 Correlation Between aPKC ${ }_{1^{\prime}}$ CD68, CD80, CD206, NF-kB, and Clinicopathologic Characteristics in 70 CCA Patients

\begin{tabular}{|c|c|c|c|c|c|c|c|c|c|c|c|c|c|c|c|c|}
\hline \multirow[t]{2}{*}{ Group } & \multirow[t]{2}{*}{$\mathrm{n}$} & \multicolumn{2}{|c|}{$\mathrm{aPKC}_{1}$} & \multirow[t]{2}{*}{$P$} & \multicolumn{2}{|c|}{ CD68 } & \multirow[t]{2}{*}{$P$} & \multicolumn{2}{|c|}{ CD80 } & \multirow[t]{2}{*}{$P$} & \multicolumn{2}{|c|}{ CD206 } & \multirow[t]{2}{*}{$P$} & \multicolumn{2}{|c|}{ NF-кB } & \multirow[t]{2}{*}{$P$} \\
\hline & & Low & High & & Low & High & & Low & High & & Low & High & & Low & High & \\
\hline \multicolumn{17}{|l|}{ Age (years) } \\
\hline$\leq 60$ & 37 & 19 & 18 & 0.208 & 12 & 25 & 0.449 & 18 & 19 & 0.602 & 19 & 18 & 0.622 & 19 & 18 & 0.316 \\
\hline$>60$ & 33 & 12 & 21 & & 8 & 25 & & 14 & 19 & & 15 & 18 & & 13 & 20 & \\
\hline \multicolumn{17}{|l|}{ Gender } \\
\hline Male & 36 & 17 & 19 & 0.611 & 10 & 26 & 0.880 & 12 & 24 & 0.089 & 16 & 20 & 0.477 & 17 & 19 & 0.794 \\
\hline Female & 34 & 14 & 20 & & 10 & 24 & & 20 & 14 & & 18 & 16 & & 15 & 19 & \\
\hline \multicolumn{17}{|c|}{ Lymphoid nodal status } \\
\hline No & 45 & 24 & 21 & 0.041 & 16 & 29 & 0.044 & 20 & 25 & 0.866 & 25 & 18 & 0.043 & 28 & 15 & $<0.001$ \\
\hline Yes & 25 & 7 & 18 & & 4 & 21 & & 12 & 13 & & 9 & 18 & & 4 & 23 & \\
\hline \multicolumn{17}{|l|}{ TNM staging } \\
\hline$|-| \mid$ & 33 & 20 & 13 & 0.009 & 14 & 19 & 0.015 & 16 & 17 & 0.819 & 24 & 9 & $<0.001$ & 25 & 8 & $<0.001$ \\
\hline III-IV & 37 & 11 & 26 & & 6 & 31 & & 16 & 19 & & 11 & 26 & & 7 & 30 & \\
\hline \multicolumn{17}{|l|}{ Differentiation } \\
\hline Well & 27 & 16 & 11 & 0.046 & 9 & 18 & 0.485 & 11 & 16 & 0.508 & 17 & 10 & 0.044 & 16 & 11 & 0.071 \\
\hline Moderate/Poorly & 43 & 15 & 28 & & 11 & 32 & & 21 & 22 & & 17 & 26 & & 16 & 27 & \\
\hline
\end{tabular}


Table 2 Univariate and multivariate analyses for predictors of overall survival

\begin{tabular}{|c|c|c|c|c|c|c|}
\hline \multirow[t]{3}{*}{ Vriables } & \multicolumn{6}{|c|}{ Overall survival } \\
\hline & \multicolumn{3}{|c|}{ Univariate analysis } & \multicolumn{3}{|c|}{ Multivariate analysis } \\
\hline & HR & $95 \% \mathrm{Cl}$ & $P$ value & $\mathrm{HR}$ & $95 \% \mathrm{Cl}$ & $P$ value \\
\hline Age $(<60$ vs $\geq 60)$ & 1.574 & $0.779-3.178$ & 0.206 & & & \\
\hline Gender (male vs female) & 0.731 & $0.371-1.440$ & 0.365 & & & \\
\hline Lymph node metastasis (no vs yes) & 5.004 & $2.258-11.087$ & $<0.001$ & 10.933 & 4.133-14.481 & 0.027 \\
\hline TNM stage (I/II vs III/IV) & 3.812 & $1.783-8.147$ & 0.001 & & & \\
\hline Differentiation (well vs moderate/poor) & 7.726 & $2.644-22.582$ & $<0.001$ & 8.368 & $2.403-29.145$ & 0.001 \\
\hline $\mathrm{aPKC}_{1}$ expression (low vs high) & 4.329 & $2.000-9.368$ & $<0.001$ & 4.766 & $1.549-14.661$ & 0.006 \\
\hline CD68 expression (low vs high) & 2.529 & $1.094-5.844$ & 0.030 & & & \\
\hline CD80 expression (low vs high) & 1.272 & $0.646-2.503$ & 0.486 & & & \\
\hline CD206 expression (low vs high) & 5.981 & $2.567-13.936$ & $<0.001$ & 3.526 & $1.268-9.808$ & 0.016 \\
\hline
\end{tabular}

HR hazard ratio, $\mathrm{Cl}$ confidence interval

and M2 macrophage infiltration are associated with chemoresistance in CCA.

To identify the effects of M2 macrophages on CCA cells, we applied a model of macrophage polarization involving the differentiation of peripheral blood mononuclear cells (PBMCs) for further analysis (Supplementary Fig. 2B). Flow cytometry and RT-PCRs were employed to analyze the phenotype of macrophages. The M2 macrophage characteristics were confirmed by the reduced expression of M1 markers (CD80 and IL-12) and the elevated expression of M2 markers (CD206 and IL-10) (Supplementary Fig. 2C-D). Consequently, we examined whether M2 macrophages could protect CCA cells from GEM chemotherapy. As shown in Fig. 2B, compared with control medium and $M \Phi-C M$ treatments, M2-CM treatment significantly reduced the sensitivity of CCA cells to GEM. Next, FACS, which was employed to detect apoptosis indicated that M2-CM treatment notably reduced the apoptosis of CCA cells induced by $10 \mu \mathrm{M}$ GEM relative to control medium and $M \Phi-C M$ treatments (Fig. 2C). Previous studies have shown that elevated aPKCı expression provides resistance to drug-induced apoptosis [19]. To verify whether $\mathrm{aPKC} \mathrm{I}$ is involved in the M2-CM-mediated protection of CCA cells against GEM-induced apoptosis, we established aPKCl-deficient CCA cell lines by transfection with aPKCl-siRNA (Supplementary Fig. 2E). WB analysis showed that the protein level of cleaved caspase-3 in cells treated with GEM plus M2-CM was markedly decreased compared to that in cells treated with GEM plus control medium. aPKCı depletion resulted in loss of the M2-CM-mediated protective effect (Fig. 2D). Interestingly, the level of phosphorylated-aPKC $\mathrm{C}_{1}$ was significantly increased in CCA cells after M2-CM treatment, while the level of $\mathrm{aPKC}_{1}$ was not affected (Fig. 2D). We further found that the IC50 value for GEM was drastically increased in aPKCt-overexpression CCA cells, but decreased in aPKCl-silenced cells (Fig. $2 \mathrm{E}$ and Supplementary Fig. 2F). Moreover, FACS was employed to validate the anti-apoptosis role of $\mathrm{aPKC}_{1}$ in CCA cells and found that upregulated aPKCı significantly reduced the GEMinduced apoptosis rates of CCA cells (Fig. 2F). However, overexpressing or silencing aPKCı only resulted in a slight change in the apoptosis rate of CCA cells without GEM treatment (Supplementary Fig. 2G). These results indicate that aPKCı may play a key role in $\mathrm{M} 2$ macrophage-induced CCA cell GEM resistance.

\footnotetext{
(See figure on next page.)

Fig. 2 M2 macrophages induce aPKCI-mediated CCA cell chemoresistance to GEM. A. Overall survival rates of CCA patients with the different expression levels of aPKC and CD206 treated with GEM-based chemotherapy or not after surgery were compared using Kaplan-Meier analysis. B. The CCK-8 assay was used to detect the sensitivity of CCA cells to GEM under different conditions: normal media, MФ-CM and M2-CM. Relative number of CCA cells treated with $10 \mu \mathrm{M}$ GEM in different conditioned media as indicated for $48 \mathrm{~h}$. C. CCA cells were incubated in different conditions as indicated in the presence or absence of $10 \mu \mathrm{M} \mathrm{GEM} \mathrm{for} 48 \mathrm{~h}$, and the percentage of apoptotic cells was analyzed by FACS. D. WB of p-aPKC and cleaved caspase-3 (WB of CCA cells transfected with scrambled control or si-aPKC $\# 1$ and treated with or without GEM in normal media or M2-CM for $48 \mathrm{~h}$ ). E. IC50 of gemcitabine in the indicated cells. Each bar represents the mean \pm SD of three independent experiments. F. Annexin V-FITC and PI staining of the indicated cells treated with gemcitabine $(10 \mu \mathrm{M})$ for $48 \mathrm{~h}$. Each bar represents the mean \pm SD of three independent experiments
} 


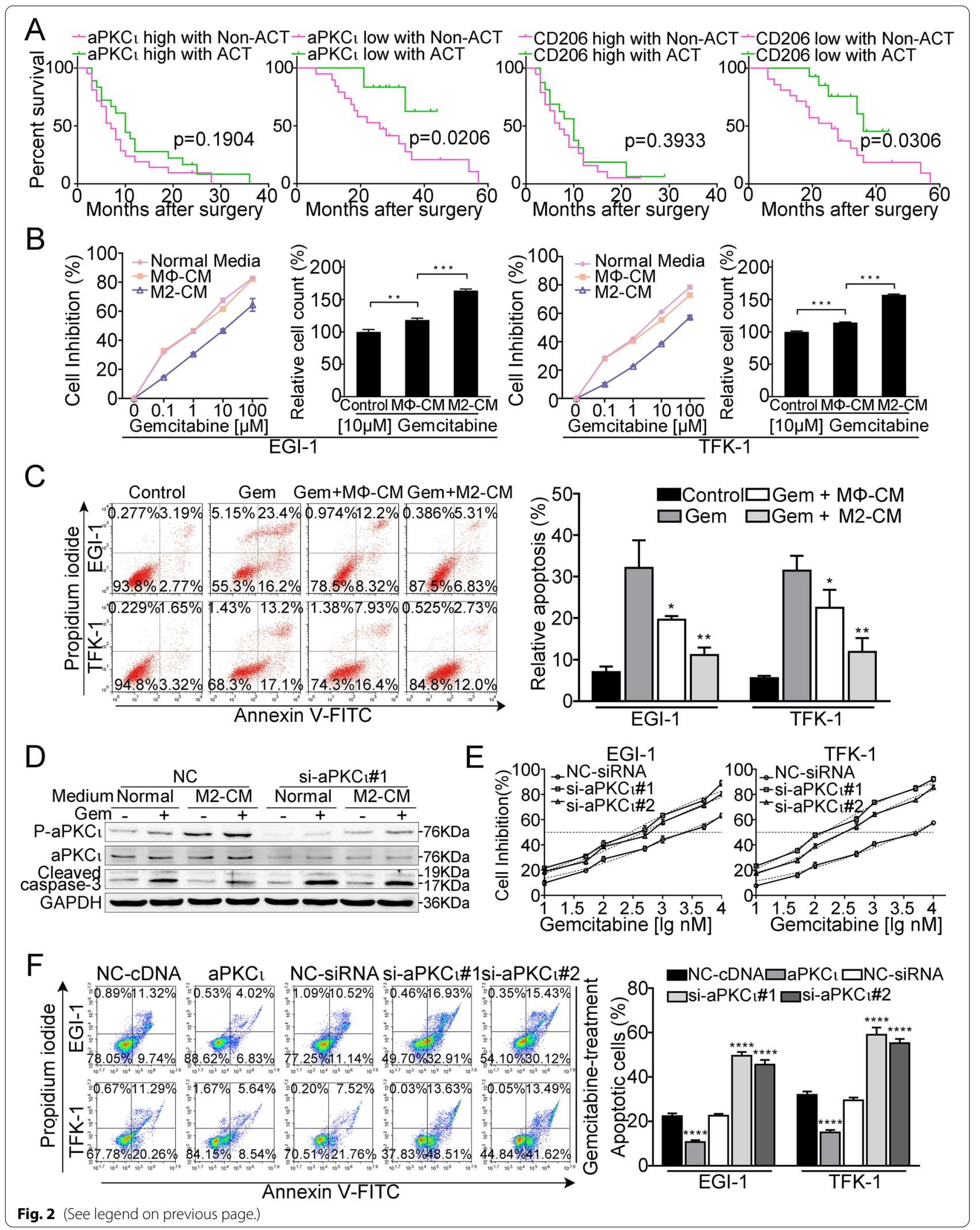




\section{aPKCı mediates NF-KB activation contributing to M2 macrophages-induced chemoresistance}

Growing evidences have demonstrated that aPKCı activates NF- $\mathrm{kB}$ signaling in multiple tumor types [20-22] and that NF- $\mathrm{kB}$ is a major transcription factor associated with the immune microenvironment $[23,24]$, chemoresistance [25] and EMT [26, 27]. To further explore the mechanism by which $\mathrm{aPKC}_{1}$ promotes CCA cells survival, we investigated the role of the NF- $\mathrm{KB}$ signaling pathway. We found that overexpressing $\mathrm{aPKC}$ induced $\mathrm{p} 65$ phosphorylation and nuclear translocation (Fig. 3A and Supplementary Fig. 3A) and enhanced NF-kB transcriptional activity (Fig. 3B). Subsequently, WB was employed to validate that $\mathrm{aPKC}$ mediates $\mathrm{NF}-\mathrm{kB}$ activation in $\mathrm{M} 2$ macrophage-induced GEM resistance. We found that $\mathrm{M} 2-\mathrm{CM}$ induced aPKCı phosphorylation and NF- $\mathrm{kB}$ activation in the presence of GEM, while $\mathrm{OPKC}_{1}$-siRNA treatment attenuated these effects (Fig. 3C). Furthermore, we assessed whether the NF- $\mathrm{kB}$ signaling was required for $\mathrm{aPKC}_{1}$-induced chemoresistance. We blocked the NF- $\mathrm{kB}$ signaling pathway by pharmacologically employing pyrrolidinedithiocarbamate ammonium (PDTC 50umol/L) or genetically exerting a dominant negative mutant of $\mathrm{I} \kappa \mathrm{B} \alpha$. Inhibition of NF- $\mathrm{KB}$ signaling enhanced the GEMinduced apoptosis rates and reduced the IC50 value of GEM in $\mathrm{aPKC}_{1}$-overexpressing CCA cells (Fig. 3D-E). Anchorage-independent growth of $\mathrm{aPKC}_{1}$-overexpressing CCA cells, treated with GEM $(10 \mu \mathrm{M})$, was suppressed in the presence of PDTC (Supplementary Fig. 3B).

Based on these findings, the clinical significance of nuclear NF-kB (P65) expression was characterized in human CCA. The IHC analysis showed that staining of nuclear NF- $\mathrm{kB}$ was enriched in CCA tissues than pair-matched paratumor tissues (Fig. 3F). Then, we analyzed the association between NF- $\mathrm{BB}$ and $\mathrm{aPKC}_{1} /$ CD206 in CCA specimens. Pearson correlation analyses of the IHC results confirmed that expression of nuclear NF-kB (P65) was significantly correlated with $\mathrm{aPKC}_{1}(\mathrm{r}=0.6992, P<0.0001)$ and CD206 $(\mathrm{r}=0.5495$, $P<0.0001$ ) (Fig. 3G). Moreover, the positive immunoreactivity of nuclear NF-kB was significantly associated with lymph node metastasis $\left(X^{2}=16.911, P<0.001\right)$ and tumor-node-metastasis (TNM) stage III-IV $\left(\chi^{2}=22.707\right.$, $P<0.001)$ as shown in Table 1 . The group with low nuclear NF- $\mathrm{kB}$ expression had better prognosis (Fig. $3 \mathrm{H}$ ). Importantly, the prognosis was also statistically associated with the co-expression of aPKCı and CD206 (Fig. 3H and Supplementary Fig. 3C). These results indicated that aPKC mediates NF- $\mathrm{kB}$ activation to contribute to M2 macrophages-induced GEM resistance.

In terms of a mechanism, previous research has confirmed that the $\mathrm{aPKC}_{1}$ could bind to P62 through a PB1$\mathrm{PB} 1$ domain interaction that regulates NF- $\mathrm{kB}$ activation [22]. Therefore, we assessed whether this mechanism exists in CCA. We performed Co-IP experiments and found that $\mathrm{aPKC}_{1}$ immunoprecipitated with $\mathrm{P} 62$, and in turn, P62 was detected in the aPKCl-immunoprecipitates (Supplementary Fig. 3D upper). To determine the molecular surfaces through which the aPKCl and P62 interaction occur, we constructed Flag-tagged wild-type and site-specific mutants ( $\mathrm{aPKC}_{\mathrm{l}}$-D72A, P62-K7A) of these proteins based on previously reported research findings [28-30]. IP experiments showed that the interaction of $\mathrm{aPKC}_{1}$ with $\mathrm{P} 62$ requires the wild-type PB1 domain, and the D72A mutation in the PB1 domain of $\mathrm{aPKC}_{1}$ abolished the interaction with P62 (Supplementary Fig. 3D bottom). In addition, WBs and luciferase reporter gene assays showed that NF- $\mathrm{kB}$ phosphorylation and transcriptional activity are regulated by $\mathrm{P} 62$. The K7A mutation in the PB1 domain of P62 abolished the WT effects (Supplementary Fig. 3E-F). This observation is similar to that of Wooten et al. [31] These results provide evidence that aPKCı may regulate NF- $\mathrm{kB}$ activation by interacting with P62.

\section{TAMs-derived TGF $\beta 1$ induce CCA cell EMT via the $\mathrm{aPKC}_{\mathrm{t}}$ and NF-KB activation}

TGF $\beta 1$ is well known to facilitate tumor formation and development [32]. Coincidentally, it is also one of the major cytokines secreted by $\mathrm{TAMs}^{5}$. Consistent with the results of the 10x Genomics single-cell sequencing technology in human cholangiocarcinoma, ELISA and qPCR showed that TGF $\beta 1$ was dramatically upregulated in intracellular and supernatant of M2

\footnotetext{
(See figure on next page.)

Fig. 3 aPKCI mediates NF-KB activation to contribute to M2 macrophages-induced chemoresistance. $\mathbf{A}$. WBs were used to detect the expression of $\mathrm{p}-\mathrm{aPKC}, \mathrm{P} 62, \mathrm{p}-\mathrm{NF}-\mathrm{KB}$ and test the NF-KB (p65) nuclear translocation in the indicated cells. CCA cells were transfected with the empty vector as a negative control (NC) and cells without any treatment were used as blank control (aPKC, ). B. NF-kB luciferase reporter activity was detected by Dual-luciferase reporter assay. C. WB for the indicated proteins of TFK-1 cells transfected with scrambled control or si-aPKC,\#1 and treated with normal media or M2-CM for $6 \mathrm{~h}$ in the absence or presence of $10 \mu \mathrm{M} \mathrm{GEM}$. D. IC50 of gemcitabine in the aPKC ${ }_{1}^{+}$cells transfected with vector, IKBa-mut, or treated with an NF-KB inhibitor (PDTC). Each bar represents the mean \pm SD of three independent experiments. E. Annexin V-FITC and PI staining of the indicated cells treated with gemcitabine $(10 \mu \mathrm{M})$ for $48 \mathrm{~h}$. F. IHC was used to examine the levels of nuclear NF-KB $(1: 200)$ in 40 paired CCA and paratumor tissues. Representative images are shown (left). Scale bar, $20 \mu \mathrm{m}$. Quantification of the nuclear NF-kB expression level in paired CCA and paratumor tissues (right). G. Linear regression was used to analyze the correlations between nuclear NF-KB with aPKC, and CD206. H. Kaplan-Meier analysis indicating the correlation between nuclear NF-KB expression and overall survival in patients with CCA (left). The overall survival curves from CCA patients with co-expression of $\mathrm{aPKC}_{1}$ and nuclear NF-KB was analyzed by Kaplan-Meier (right)
} 


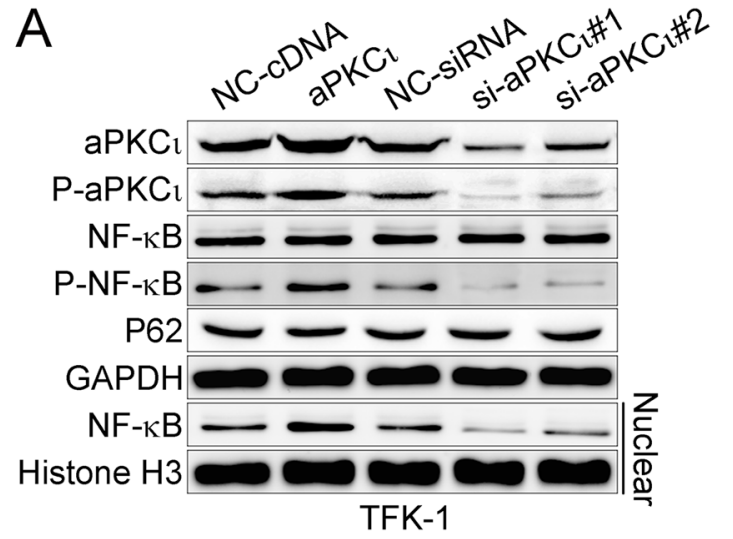

C

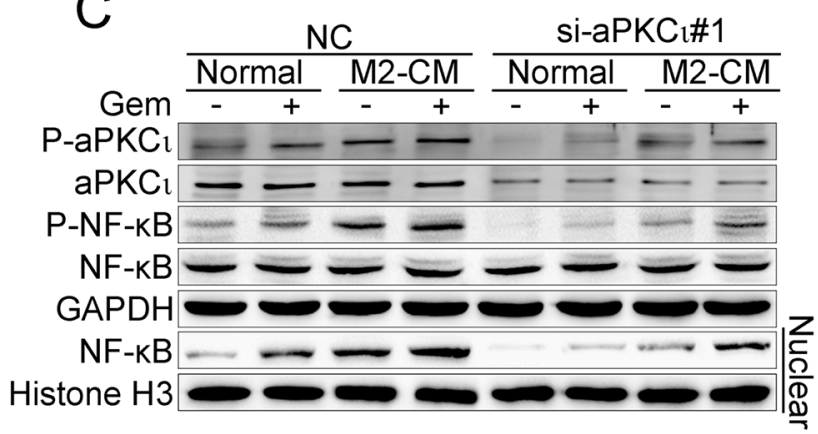

E

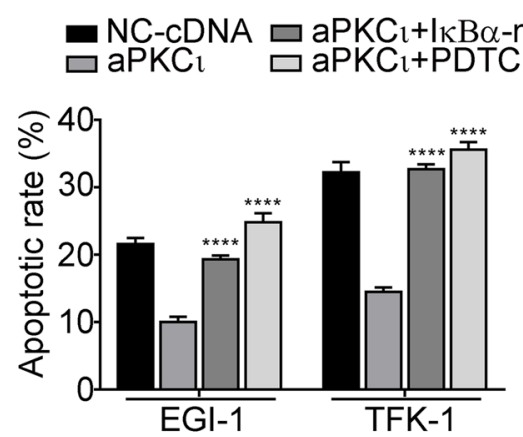

$G$

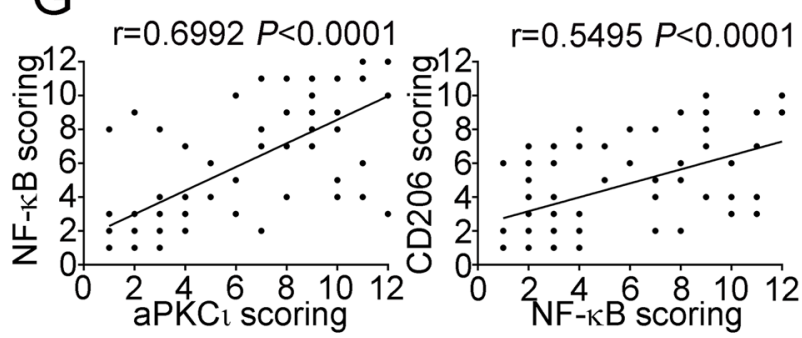

Fig. 3 (See legend on previous page.)

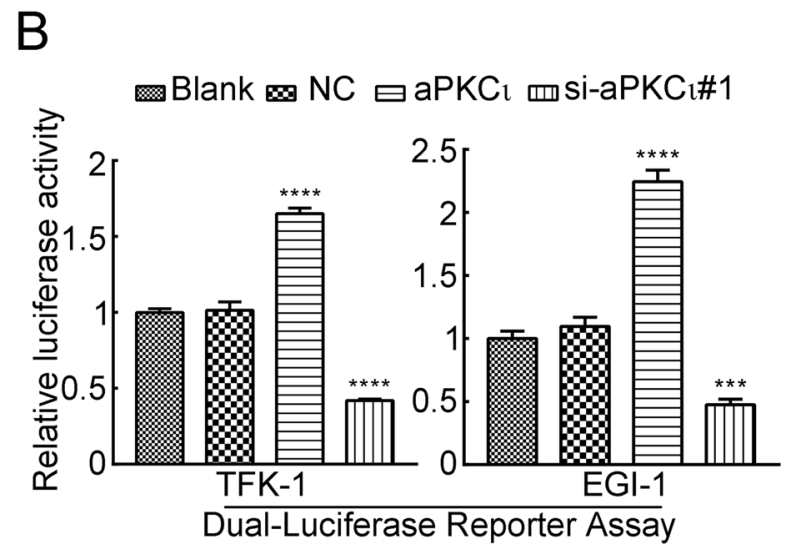

$\mathrm{D}$

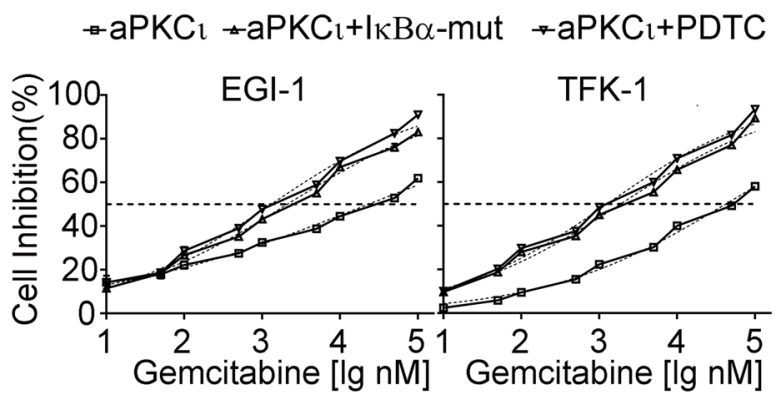

F
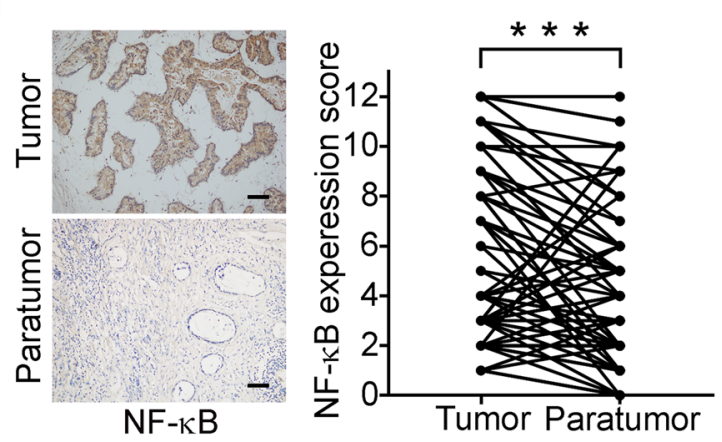

$\mathrm{H}$

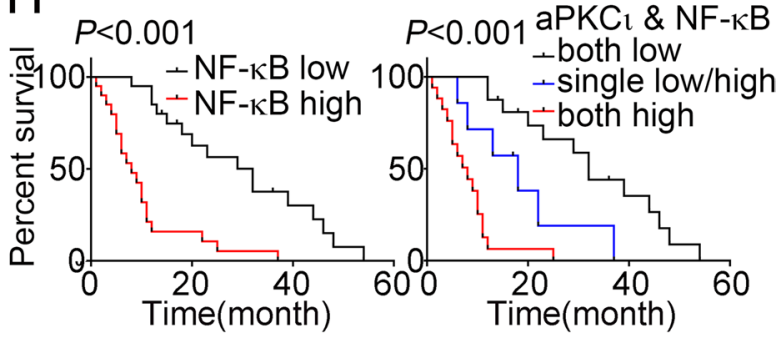

macrophages compared with M $\mathrm{M} / \mathrm{M} 1$ macrophages (Supplementary Fig. 4A). Therefore, we hypothesized that M2 macrophages-derived TGF $\beta 1$ might contribute to the promotion of CCA cell EMT. We have previously demonstrated the critical role of $\mathrm{aPKC}_{1}$ in TGF $\beta 1$-induced EMT in CCA cells [33]. Here, we found that TGF $\beta 1$ induced $\mathrm{aPKC}_{1}$ and NF- $\mathrm{NB}$ phospho-activation in a time-dependent manner, whereas $\mathrm{aPKC}_{1}$-siRNA 
treatment attenuated the effects (Fig. 4A). Moreover, the levels of $\mathrm{p}-\mathrm{aPKC}$ and $\mathrm{p}-\mathrm{NF}-\mathrm{kB}$ were increased in ${ } \mathrm{PKC}_{1}$-transduced CCA cells treated with M2-CM, while anti-TGF $\beta 1$ neutralizing antibody or LY2157299 (a selective TGF $\beta$ receptor inhibitor) treatment reversed the above effects (Fig. 4B). Similarly, we found that TGF $\beta 1$ induced NF- $\mathrm{kB}$ transcriptional activity and nuclear translocation, and this response was dependent on $\mathrm{aPKC}_{\mathfrak{v}}$, because $\mathrm{APKC}_{1}$ depletion through siRNA resulted in loss of the effect (Fig. 4C-D and Supplementary Fig. 4B).

We have found that $\mathrm{aPKC}_{1}$ induces EMT-like protein expression [15] (Supplementary Fig. 4C), it was not surprising to see that the morphology of CCA cells treated with M2-CM changed from a broad elliptical shape to a long fusiform shape. The M2-CM-treated CCA cells had reduced expression of E-cadherin and increased expression of vimentin, which facilitates migration and invasiveness. However, anti-TGF $\beta 1$ neutralizing antibody or LY2157299 treatment suppressed the above EMT changes, TGF $\beta 1$ treatment alone acted as a positive control, suggesting that M2 macrophages-derived TGF $\beta 1$ induced CCA cell EMT (Fig. 4E-F and Supplementary Fig. 4D-E). Meanwhile, to further investigate whether NF- $\mathrm{kB}$ activation was essential for M2 macrophagesderived TGF $\beta 1$-induced CCA cells EMT, we employed PDTC to block the NF-kB signaling pathway. Strikingly, the inhibition caused reversal of TGF $\beta 1$-induced EMT, including up-regulation of E-cadherin, down-regulation of vimentin, as well as decreased capacity of cell invasion and migration (Fig. 4E-F and Supplementary Fig. 4D-E). Collectively, these data suggested that TAMs-derived TGF $\beta 1$ induces CCA cell EMT via the $a \mathrm{PKC}_{\mathrm{l}} / \mathrm{NF}-\mathrm{kB}$ pathway.

\section{CCL5 secreted by aPKCI-induced mesenchymal-like CCA cells mediates the chemotactic migration and activation of macrophages}

To investigate whether mesenchymal-like CCA cells activate macrophages, we cocultured mesenchymallike CCA cell lines by aPKCı transfection with PBMCsinduced macrophages. Flow cytometry showed that the proportions of M2 macrophages were significantly increased compared to macrophages cultured with the control group (Supplementary Fig. 5A). Moreover, cell migration assay revealed that CCA cells with $\mathrm{aPKC}_{1}$ overexpression promote the $\mathrm{CD} 14+$ monocytes recruitment (Supplementary Fig. 5B). To understand how aPKClinduced mesenchymal-like CCA cells exert their functions, we employed a human inflammation antibody array to identify the profile of cytokines secreted by mesenchymal-like TFK-1 cells. Interestingly, CCL5, which is a target gene of NF- $\mathrm{KB}[34]$ and an established chemoattractant for macrophages [35], was found among these cytokines (Fig. 5A). In agreement, ELISA, qRT-PCR and WB assays confirmed the increase of CCL5 production in mesenchymal-like CCA cells mediated by aPKCr (Fig. 5B and Supplementary Fig. 5C). Meanwhile, we found that NF-kB inhibition through PDTC resulted in loss of the aPKCl-mediated effect (Fig. 5B). To assess whether TGF $\beta 1$ and NF- $\mathrm{KB}$ transcription factors activate CCL5 promoter activity, luciferase reporter gene assays were performed using a CCL5 promoter with a mutated NF- $\mathrm{KB}$ binding site (Supplementary Fig. 5D). As expected, TGF $\beta 1$ treatment increased the transcriptional activity of CCL5, and the NF- $\mathrm{KB}$ binding site mutation in the CCL5 promoter attenuated this effect (Fig. $5 \mathrm{C}$ ). On the basis of our data, we speculate that aPKCl/NF-kB/CCL5 signaling is involved in the macrophages recruitment and activation. To further verify our hypothesis we could show that CCL5 treatment enhanced M2 macrophage polarization and $\mathrm{CD} 14^{+}$monocytes recruitment, whereas targeting CCL5 with neutralizing antibodies potently abrogated the effects. Moreover, we also found that PDTC treatment attenuate these effects (Fig. 5D-E and Supplementary Fig. 5E). These findings indicated that TGF $\beta 1$ could induce the expression and secretion of CCL5 in CCA to mediate the chemotactic migration and activation of macrophages by $\mathrm{aPKC}_{1} / \mathrm{NF}-\mathrm{k} B$ signaling pathway.

Finally, we performed WB to analyze the mechanism of macrophages activation, and several classical signaling pathways involved in macrophage functions were evaluated. The results suggest that the activation of STAT3 signaling may be involved in CCL5-mediated macrophage recruitment and M2 polarization (Supplementary Fig. 5F).

\section{The macrophage-aPKC,-CCL5 feedback loop promotes CCA growth and metastasis in vivo}

To investigate the crucial role of TAMs in the aPKClmediated progression of CCA in vivo, we established a xenograft model and lung metastasis model of human CCA according to the schematic shown in Fig. 6A and $D$. We found that the growth of xenografts was inhibited (Fig. 6B and Supplementary Fig. 6A) and the number of metastatic nodules was reduced after macrophages were selectively depleted (Fig. 6E). Notably, the depletion of macrophages resulted in a reduction in $\mathrm{p}$-aPKCı expression (Fig. 6C, F and Supplementary Fig. 6B). These results suggest that the effect of TAMs on CCA progression in vivo may be mediated by the aPKCı signaling pathway. To further investigate the mechanism by which aPKC। regulates CCA development and the interaction between mesenchymal-like CCA cells and macrophages, we stably upregulated aPKCı expression in CCA cell lines. The macrophages recruitment experiment (Fig. 6G) demonstrated that $\mathrm{aPKCl}$ overexpression promoted tumor 


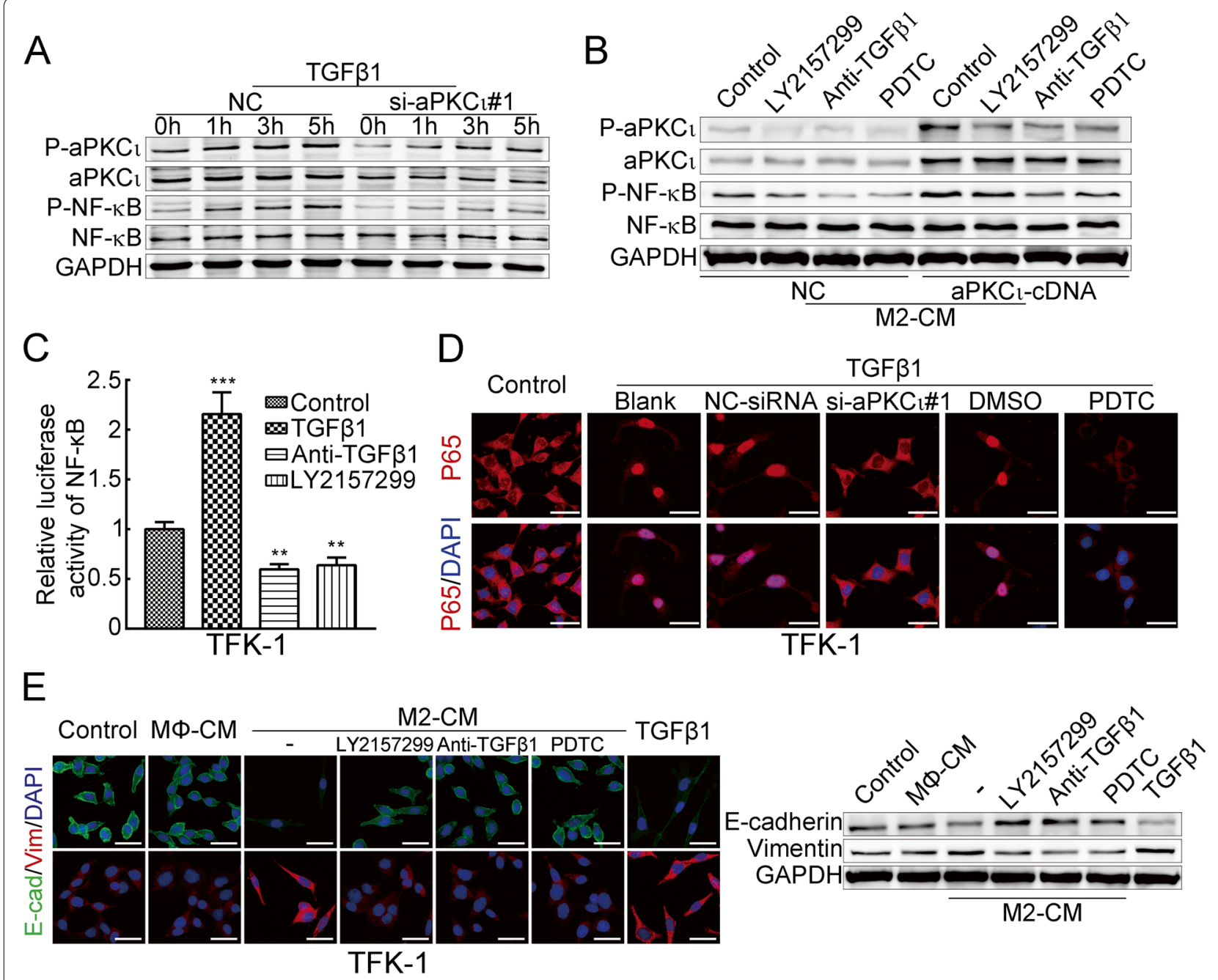

$\mathrm{F}$

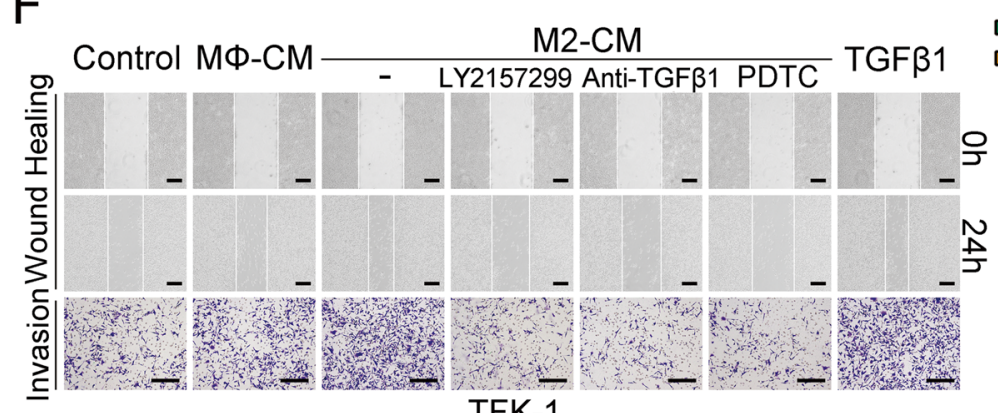

$\square$ Control $\square \mathrm{M} \Phi-C M \square M 2-C M \square M 2-C M+L Y 2157299$ $\square \mathrm{M} 2-\mathrm{CM}+$ Anti-TGFß1 $\square \mathrm{M} 2-\mathrm{CM}+\mathrm{PDTC} \square \mathrm{TGF}$ 1 จㅇ

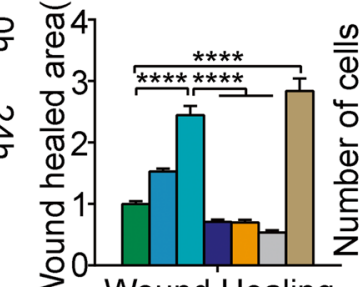

Wound Healing

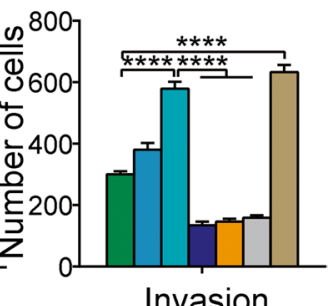

Fig. 4 TAMs-derived TGFB1 induce CCA cell EMT via the $\mathrm{APKC}$, and NF-KB activation. A. WBs was used to detect the activation of aPKC, and NF- $\mathrm{B} B$ in CCA cells transfected with control-siRNA or aPKC-siRNA after TGF $\beta 1$ treatment for the indicated times. $\mathbf{B}$. WBs were employed to detect the activation of $\mathrm{aPKC}_{1}$ and NF-KB in CCA cells transfected with $\mathrm{aPKC} \mathrm{C}_{1} \mathrm{CDNA}$ or control-cDNA after treatment with CM from $\mathrm{M} 2$ macrophages alone or those treated with a TGF $\beta R 1$ inhibitor (LY2157299), an anti-TGF 1 1 neutralizing antibody, or pyrrolidinedithiocarbamate ammonium (PDTC). C. NF-KB luciferase reporter NF-KB activity in the indicated cells, either treated with TGF $\beta 1$ for $5 \mathrm{~h}$ or treated with or without LY2157299 $(2 \mu \mathrm{M})$ or a neutralizing anti-TGF $\beta 1$ antibody $(2 \mu \mathrm{g} / \mathrm{mL})$ for $5 \mathrm{~h}$. D. Confocal fluorescence microscopy of p65/DAPI staining in TFK-1 cells treated with or without TGF $\beta 1$ together with PDTC or aPKC,-siRNA treatment. Scale bar, $20 \mu \mathrm{m}$. E. Immunofluorescence and WB were used to detect E-cadherin and vimentin expression in CCA cells treated with or without CM from MФ macrophages (MФ-CM), M2-CM alone or with LY2157299, an anti-TGF $\beta 1$ neutralizing antibody, PDTC, or recombinant human TGF $\beta 1$. Scale bar, $20 \mu \mathrm{m}$. F. Wound healing and invasion assays of CCA cells treated as described in (E). Scale bar, $200 \mu \mathrm{m}$ (mean $\pm \mathrm{SD}, n=3 ;{ }^{*} P<0.05,{ }^{* *} P<0.01,{ }^{* * *} P<0.001$ and ${ }^{* * * *} P<0.0001 ; P$ values were obtained using two-tailed Student's $\mathrm{t}$ tests) 


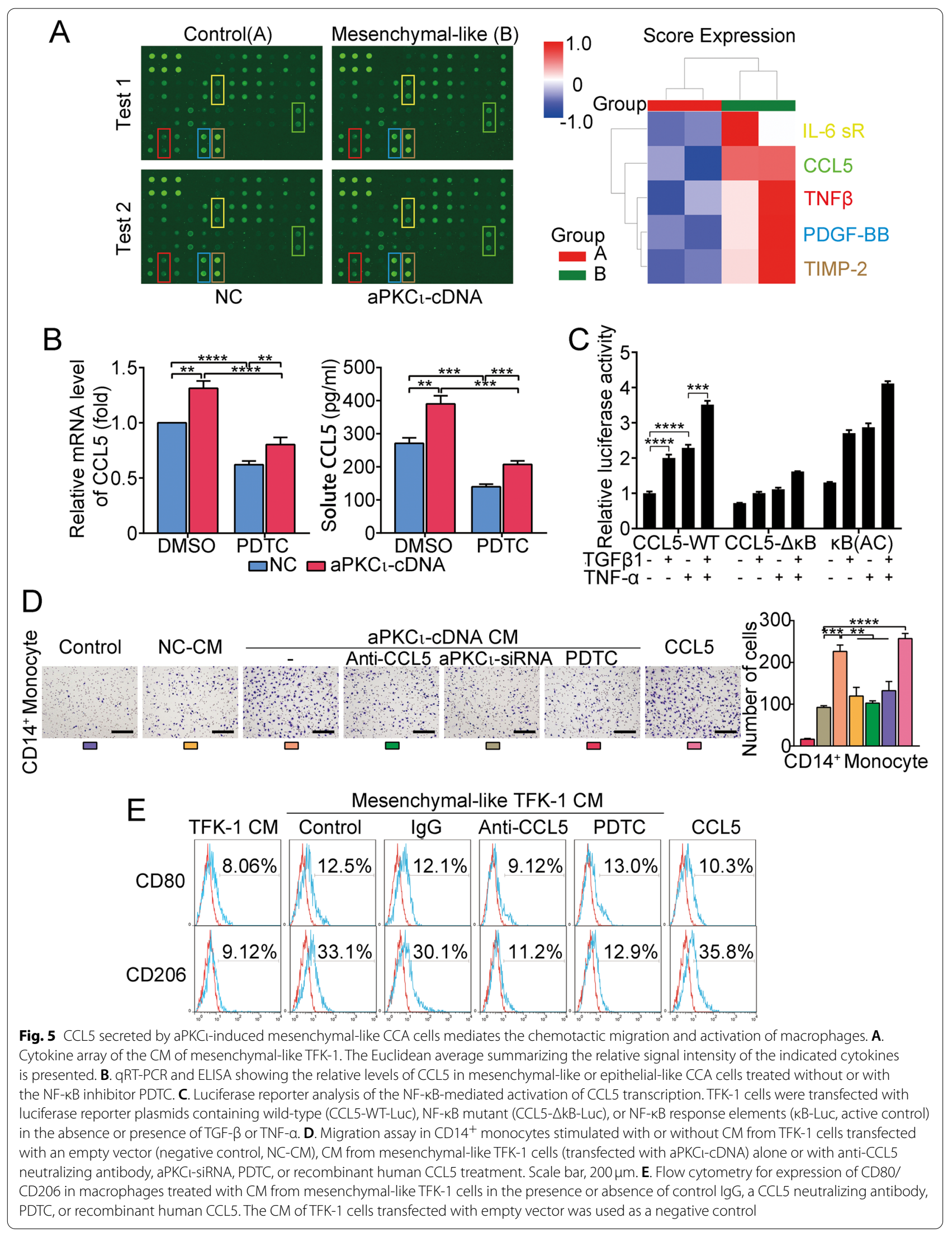


progression (Fig. 6H and Supplementary Fig. 6C). Furthermore, the number of $\mathrm{F} 4 / 80^{+}$macrophages in aPKClderived xenografts was higher than that in the negative control xenografts (Fig. 6I and Supplementary Fig. 6D). Because the CCL5-CCR5 axis is a major regulator of macrophage recruitment [36, 37], we investigated the role of aPKCl/CCL5 pathway in regulating macrophage recruitment in vivo. There was a greater infiltration of $\mathrm{CCR}^{+}$macrophages in aPKCl-derived tumors than control tumors, and antagonizing the CCL5-CCR5 axis significantly reversed this effect and decreased tumor development. These findings suggest that the effects of aPKCı overexpression on CCA progression are dependent on CCL5-mediated macrophage infiltration. Importantly, we observed that the reduction in macrophage recruitment potently inhibits the expression of $\mathrm{p}-\mathrm{aPKCl}$ in CCA xenografts (Fig. 6I and Supplementary Fig. 6D). Combined with all the in vitro results, these findings suggest that aPKCl-induced CCL5 from mesenchymal-like CCA cells and TGF $\beta 1$ from TAMs form a positive feedback loop and promote CCA progression, and that aPKCı plays a key role in this process.

\section{Co-delivery of aPKCI-siRNA and GEM via liposomes for the effective treatment of CCA}

All liposomes were prepared as shown in Materials and Methods. Then, they were characterized to ensure that they qualified as liposomes. The key physicochemical characteristics of GEM-L and GEM-aPKCl-siRNA-L are shown in Supplementary Fig. 7. A soft agar growth assay was employed to evaluate the inhibitory effect of the various formulations. Compared to other treatment groups, the GEM-aPKCi-siRNA-L group showed significant inhibition of CCA cells anchorage-independent growth (Fig. 7A). To further evaluate the antitumor efficacy of the various formulations, we established a xenograft model of human CCA according to the schematic shown in Fig. 7B. Notably, consistent with the in vitro findings, Gem-aPKCl-siRNA-L-treated tumors had the smallest sizes among those of all treatment groups (Fig. 7C). To demonstrate the key role of aPKCl-siRNA in the inhibition of tumor growth, we sectioned the xenografts and analyzed the expression of aPKCl. We found that aPKCl-siRNA effectively interfered with aPKCı protein expression in vivo only when it was delivered effectively by liposomes. Furthermore, we observed a significant decrease in the number of $\mathrm{F} 4 / 80^{+}$macrophages when aPKCı was effectively knocked down (Fig. 7D). In addition, we found that treatment with GEM increased NF-kB expression (Fig. 7D). These data preliminarily suggest that the co-delivery of aPKCl-siRNA and GEM via liposomes produces enhanced antitumor effects in CCA.

Together, our results show that the Macrophages${ }_{\mathrm{aPKC}}$-CCL5 feedback loop between mesenchymal-like cancer cells and TAMs promotes CCA progression and chemoresistance. Moreover, the liposome-encapsulated aPKCl-siRNA and GEM antagonized CCA GEM chemoresistance (Fig. 7E and F).

\section{Discussion}

CCA is an aggressively invasive tumor with drug resistance and poor prognosis. The highly desmoplastic microenvironment of CCA presents an intricate immunological system [9]. Recent data have shown that cancer cells and infiltrating immune cells interact with each other by contacting or secreting cytokines to modulate the response to chemotherapy [38]. Understanding this interaction may offer new strategies for oncotherapy. Although CCA patients with TAM infiltration have poor long-term survival, the underlying mechanisms remain vague due to the limited number of studies. Thus, we investigated the crosstalk between CCA cells and TAMs and sought to identify a potential strategy for the remission of chemoresistance.

To study the mechanisms, we verified that increased expression of CD68 and CD206 in CCA tissue was positively correlated with poor outcomes. Several studies have demonstrated that $\mathrm{aPKCl}$ is involved in cell survival and plays a protective role against apoptotic stimuli $[19,39,40]$. Furthermore, NF-KB has been reported to be abnormally expressed in CCA cells and has been shown to be linked to malignant aggressiveness and chemoresistance through the release of proinflammatory cytokines $[41,42]$. Notably, we found that the correlation between $\mathrm{aPKCl}$ and the expression of NF- $\mathrm{KB}$, CD68, and CD206 is positive, and this biomarker combination is more effective than single biomarkers for predicting survival outcomes in patients with CCA. Thus,

(See figure on next page.)

Fig. 6 The macrophage-aPKC - -CCL5 feedback loop promotes CCA growth and metastasis in vivo. A. Schematic for macrophage depletion in human CCA cell xenografts. B. The volume (left) and representative images (right) of xenograft tumors are shown from two different groups treated with clodronate liposomes or control liposomes. C. Representative images from tumor sample serial sections stained for aPKC ${ }_{v}, \mathrm{P}^{-a P K C_{1}}$ and F4/80 are shown. Scale bar, $200 \mu \mathrm{m}$. D. Schematic for macrophage depletion in the lung metastasis models. $\mathbf{E}$. The number of lung metastatic nodules was evaluated from different groups treated with or without clodronate liposomes. F. Haematoxylin-eosin (HE; left) and IHC (middle and right) were used to illustrate the expression of F4/80 and p-aPKC, in lung metastatic nodules. Scale bar, $200 \mu \mathrm{m}$. G. Schematic for macrophage recruitment in human CCA cell xenografts. $\mathbf{H}$. The volume (upper) and representative images (bottom) of xenograft tumors are shown from three different groups as indicated. I. Representative images from xenograft tumor serial sections stained for aPKC ${ }^{\prime}$ CCR5, p-aPKC 1 and F4/80 are shown. Scale bar, $200 \mu \mathrm{m}$ 


\section{A} Subcutaneous xenografts
of Human CCA cells or Control-L intravenously transfected with aPKC $1-\mathrm{CDNA}$ twice a week

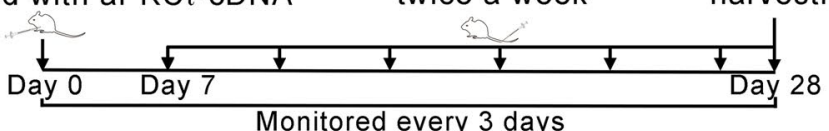

$\mathrm{B}$

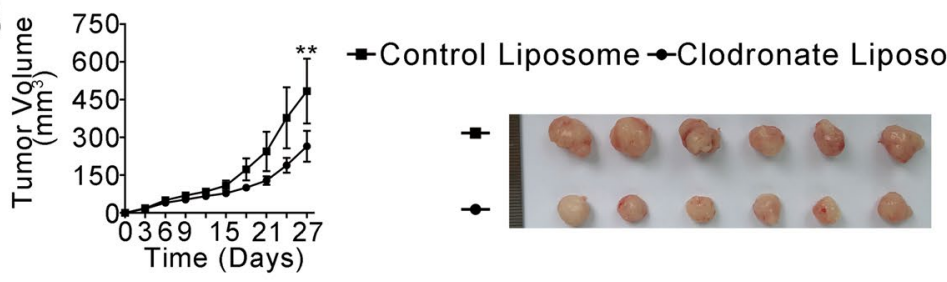

C

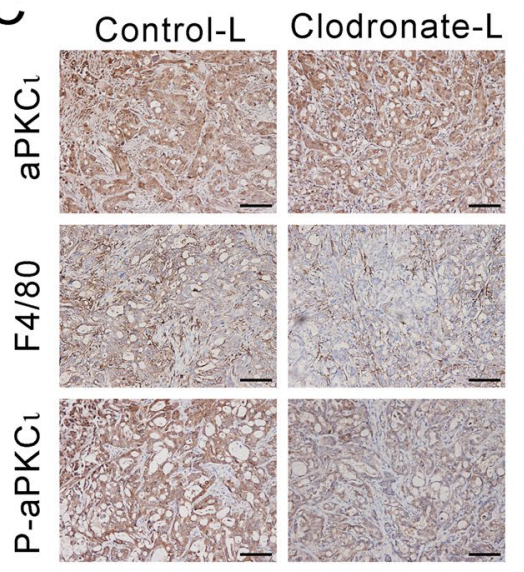

Sacrifice

for Lung

harvesting $\begin{array}{cc}\begin{array}{c}\text { Injecting EGI-1cells } \\ \text { transfected with aPKC } 1-c D N A \\ \text { into tail of mice }\end{array} & \begin{array}{c}\text { Injected with Clodronate-L } \\ \text { or Control liposomes }\end{array} \\ \text { intravenously twice a week }\end{array}$ $\begin{array}{cc}\begin{array}{c}\text { Injecting EGI-1cells } \\ \text { transfected with aPKC } 1-c D N A \\ \text { into tail of mice }\end{array} & \begin{array}{c}\text { Injected with Clodronate-L } \\ \text { or Control liposomes }\end{array} \\ \text { intravenously twice a week }\end{array}$ Day 0 Day 7 D

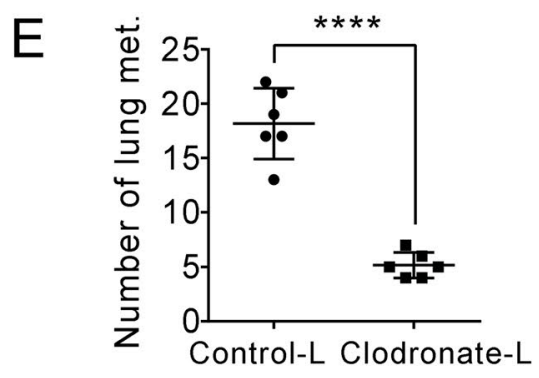

$G$ Subcutaneous Treatment with Maraviroc Sacrifice xenografts of Human or vehicle by oral for tumor CCA cells

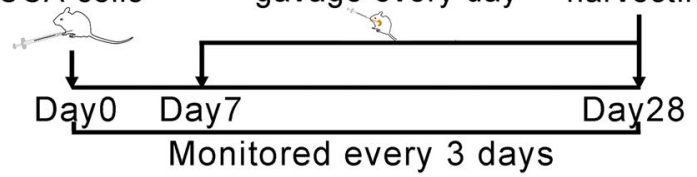

$\mathrm{H}$

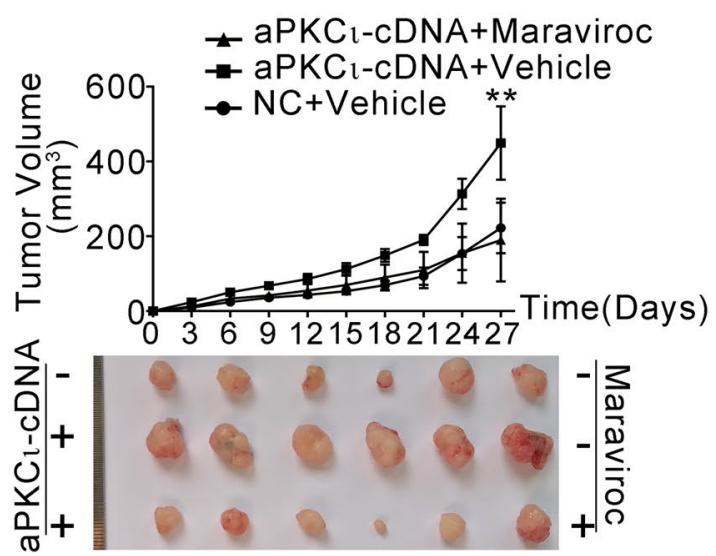

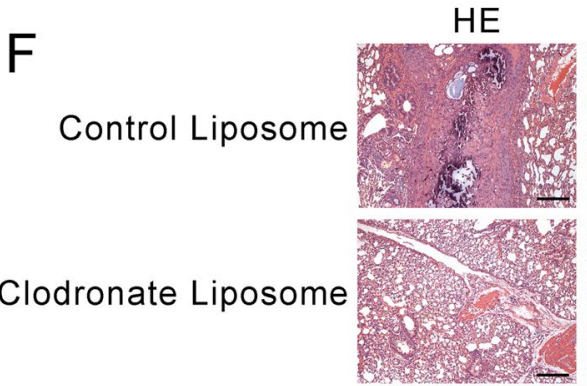

$\mathrm{F} 4 / 80$

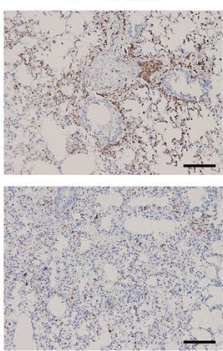

$\mathrm{P}-\mathrm{aPKC} 1$

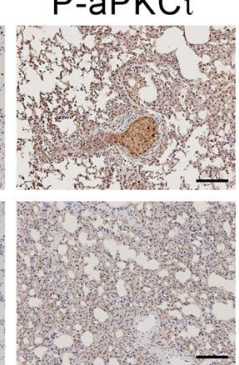

\section{I}

(n)

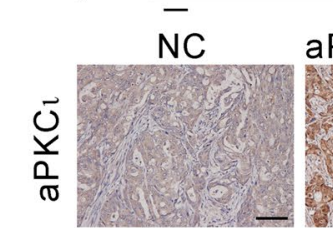

Maraviroc

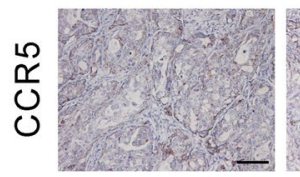

aPKC $_{1-c D N A}$ aPKC 1 -cDNA

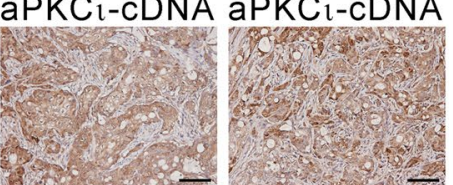

$\frac{\vec{U}}{\frac{\pi}{\pi}}$

a
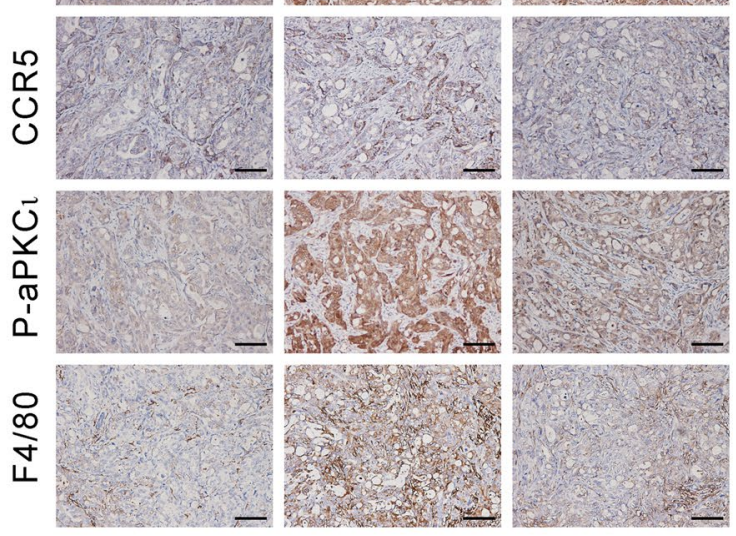

Fig. 6 (See legend on previous page.) 
we hypothesized that aPKCı may play an essential role of shaping interactions between cancer cells and their associated macrophages via NF- $\mathrm{kB}$ signaling, which results in chemoresistance.

To further confirm our hypothesis, we constructed monocyte-derived macrophages (MDMs) generated from peripheral blood monocytes (PBMCs) and polarized them into M2 macrophages that are defined as TAMs in a variety of cancers $[6,43]$. Here we found that TAMs induce GEM resistance by inhibiting apoptosis. A similar conclusion has been reported for pancreatic adenocarcinoma [44]. In the present study, we provide direct evidence for the activation of the NF- $\mathrm{kB}$ pathway by $\mathrm{aPKC}_{1} / \mathrm{P} 62$ signaling in CCA. This result broadly confirms the work of other studies in this area linking aPKCı to $\mathrm{P} 62$ via the PB1 domain $[29,45]$.

The EMT process is executed in the tumor invasive front [14], where TAMs are usually located [46]. These reports suggest that cancer cells undergoing EMT may have advantages in shaping interactions between cancer cells and their associated macrophages. Indeed, our study found that TGF $\beta 1$ from TAMs induced EMT in CCA cells. Additionally, aPKC - -siRNA treatment restrained NF- $\mathrm{kB}$ nuclear translocation and dampened the EMT response induced by TAMs. Subsequently, we selectively depleted macrophages in vivo, to demonstrate that TAMs play an important role in tumor progression. The use of CSF1R inhibitors is another appealing therapeutic strategy to target TAMs but has limited antitumor effects [47].

In turn, to further explore the mechanism of how CCA cells undergoing EMT regulate TAMs, we performed a cytokine array to identify cytokines secreted by mesenchymal-like cells overexpressing $\mathrm{aPKC}_{1}$. Notably, among the cytokines, CCL5 was apparently induced. CCL5 is known to be a key factor in the tumor microenvironment, especially in recruiting monocytes and promoting macrophage function $[8,35,36]$. The experiments in vitro and vivo demonstrated that $\mathrm{aPKC}_{1}$-CCL5-CCR5 axis recruits macrophages and programs their function of promoting tumor progression.

Collectively, these experiments demonstrate that TAMs induce $\mathrm{aPKC}_{1}$ phosphorylation by producing TGF $\beta 1$, which induces EMT and chemoresistance. In turn, CCA cells undergoing EMT secrete CCL5, which recruits and activates macrophages, constituting a Macrophages-aPKC - CCL5 positive feedback loop. Consistent with our results, several positive feedback loops between cancer cells and TAMs, such as the GM-CSFCCL18 loop, which promotes metastasis [48], and the CXCL1/2-S100A8/9 loop, which causes chemoresistance [49], have been reported. Currently, our understanding of the molecular mechanisms altering macrophage polarization is fragmented and incomplete. This is a meaningful topic for future research. Taken together, these data show that the Macrophages-aPKC ${ }_{1}$-CCL5 loop could be a potential therapeutic target for CCA treatment, especially in cancers with high $\mathrm{PPKC}_{1}$ expression levels.

Despite aPKC ${ }_{1}$-siRNA exhibiting an anticancer effect in CCA cell lines, its therapeutic efficacy has not been assessed under translational medicine circumstances. Our data confirmed that the overexpression and activation of $\mathrm{aPKC}_{1}$ can facilitate GEM resistance and cell survival in CCA. Thus, we sought to combine aPKC siRNA and GEM to explore the anticancer effect. Due to the inefficient cell uptake and biological instability of nucleic acids, we designed liposomes that can simultaneously deliver $\mathrm{aPKC}_{1}$-siRNA and GEM. Subsequently, we demonstrated that co-deliver of $\mathrm{aPKC}_{1}$-siRNA and GEM by liposomes exhibits enhanced anti-tumor effects in vitro and in vivo. Unfortunately, because this is a preliminary investigation, we did not investigate the toxicity, pharmacokinetics, cellular uptake and biodistribution of the liposomes. In addition, previous studies have suggested that liposomes are associated with toxicity and inflammatory responses. Although assorted modifications have been attempted to achieve a balance between gene delivery toxicity and efficacy, vector-induced toxicity is still a challenge for nanoparticles. More broadly, research is still needed to improve the transfection efficiency and reduce toxicity. Notwithstanding these limitations, this study partially substantiates the rationality of the co-delivery of GEM and $\mathrm{aPKC}_{1}$ siRNA since GEM-aPKC ${ }_{1}$-siRNA-L presented enhanced synergistic antitumor effects in vivo. This exploration would be fruitful for further work to improve the anticancer effect in CCA.

\footnotetext{
(See figure on next page.)

Fig. 7 Co-delivery of aPKCI-siRNA and GEM via liposomes for the effective treatment of CCA. A. Anchorage-independent growth of CCA cells in soft agar in the presence of si-NC-L, aPKCI-siRNA-L, GEM, GEM-aPKCI-siRNA, GEM-L, GEM-aPKCI-siRNA-L compared with that of cells in the presence of PBS alone. B. Schematic demonstrating the method for the analysis of the antitumor effect in human CCA cell xenografts. $C$. The tumor volume (left) and representative images (right) of xenograft tumors are shown from different groups. Data represent the mean \pm SEM. ${ }^{*} P<0.05$, ${ }^{* * *} P<0.001$. D. Immunohistochemical staining of aPKCI, F4/80 and NF-KB protein in tumor tissue in various treatment groups. Scale bar, $200 \mu \mathrm{m}$. E. The structure and antitumor mechanisms of GEM-aPKCI-siRNA-L. F. Schematic illustration of the proposed Macrophages-aPKC,-CCL5 feedback loop involved in the regulation of cholangiocarcinoma progression and improvement in chemotherapeutic responses
} 


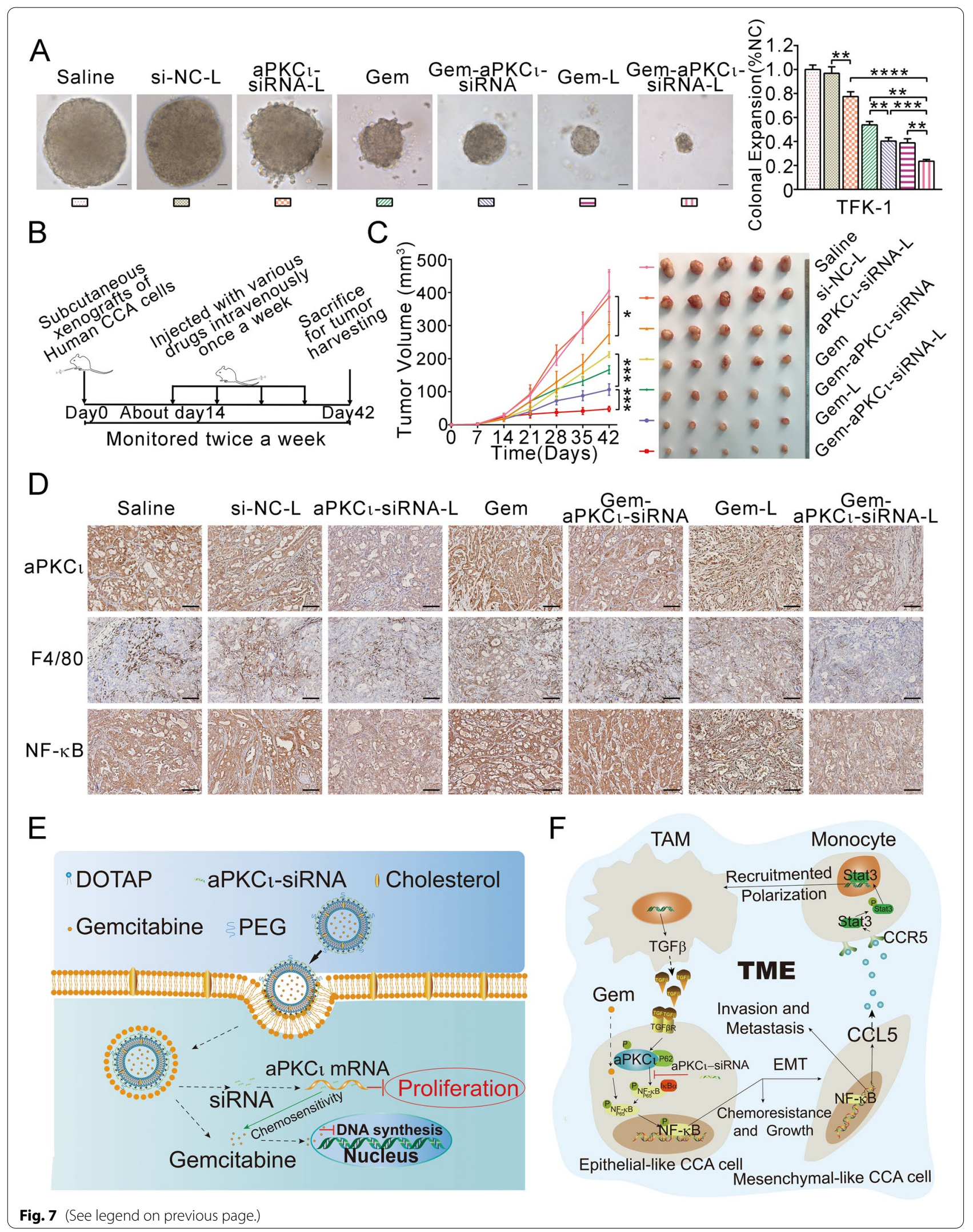




\section{Conclusions}

In summary, we demonstrated the prognostic significance of macrophages and $\mathrm{aPKC}_{1}$ in $\mathrm{CCA}$, and then identified the Macrophages-aPKC - -CCL5 positive feedback loop in the tumor microenvironment that accelerates CCA progression and chemoresistance. Finally, we designed biocompatible liposomes to co-deliver GEM and $\mathrm{aPKC}_{1}$-siRNA for CCA treatment and confirmed their synergistic antitumor effects. This thesis may deepen our understanding regarding the role of $\mathrm{aPKC}_{1}$ in the CCA microenvironment and provides an important basis for the exploitation of new, effective therapeutic strategy for CCA.

\section{Abbreviations}

CCA: Cholangiocarcinoma; aPKC : Atypical protein kinase C iota; EMT: Epithelial-mesenchymal transition; TAMs: Tumor-associated macrophages; CM: Conditioned medium; GEM: Gemcitabine; TME: Tumor microenvironment; PBMCs: Peripheral blood monocytes; PDTC: Pyrrolidinedithiocarbamate ammonium.

\section{Supplementary Information}

The online version contains supplementary material available at https://doi. org/10.1186/s13046-021-02235-8.

\section{Additional file 1. \\ Additional file 2. \\ Additional file 3. \\ Additional file 4. \\ Additional file 5. \\ Additional file 6.}

Additional file 7.

Additional file 8.

\section{Acknowledgements}

Not applicable.

\section{Authors' contributions}

T.Y., Z.-D. D conceived the idea and designed the study, performed most of the experiments, analyzed data, and wrote the manuscript. T. Y. designed and synthesized the liposomes. L. X., X.-Y L, Y.-W. Q., W. Y., Y. L., L. T. provided help for in vivo and in vitro experiments. J.-M.W. and Y.-W. supervised the entire project. The author(s) read and approved the final manuscript.

\section{Funding}

The study was supported by National Natural Science Foundation of China (No.81874062, 82072730), Translational Medicine Research Project of Tongj Hospital (No.2016ZHYX17) and Youth Program of National Natural Science Foundation of China (81902439).

\section{Availability of data and materials}

The datasets generated and/or analyzed during the current study are not publicly available due to ethical reasons but are available from the corresponding author on reasonable request.

\section{Declarations}

\section{Ethics approval and consent to participate}

The authors declare that the collection of tissue samples and clinicopathological data was approved by the Tongji Hospital Research Ethics Committee. The authors declare that the mice were cared for in strict accordance with the institutional guidelines for animal care and approved by the Committee on the Ethics of Animal Care and Use.

\section{Consent for publication}

All authors have agreed to publish this manuscript.

\section{Competing interests}

The authors declare that they have no competing interests.

\section{Author details}

${ }^{1}$ Department of Biliary and Pancreatic Surgery/Cancer Research Center Affiliated Tongji Hospital, Tongji Medical College, Huazhong University of Science and Technology, Wuhan 430030, Hubei, China. ${ }^{2}$ Department of Hepatobiliary Surgery, Renmin Hospital of Wuhan University, Wuhan 430060, Hubei, China. ${ }^{3}$ School of Pharmacy, Tongji Medical College, Huazhong University of Science and Technology, Wuhan 430022, Hubei, China. ${ }^{4}$ Department of General Surgery, Jiangsu Province Hospital and Nanjing Medical University First Affiliated Hospital, Nanjing 210009, Jiangsu, China. ${ }^{5}$ Department of Oncology Affiliated Tongji Hospital, Tongji Medical College, Huazhong University of Science and Technology, Wuhan 430030, Hubei, China. ${ }^{6}$ Affiliated Tianyou Hospital, Wuhan University of Science \& Technology, Wuhan 430064, China.

Received: 23 September 2021 Accepted: 26 December 2021 Published online: 15 January 2022

References

1. Razumilava N, Gores GJ. Cholangiocarcinoma. Lancet. 2014;383:2168-79.

2. Blechacz B, Komuta M, Roskams T, Gores GJ. Clinical diagnosis and staging of cholangiocarcinoma. Nat Rev Gastroenterol Hepatol. 2011;8:512-22.

3. Banales JM, Cardinale V, Carpino G, Marzioni M, Andersen JB, Invernizzi P, et al. Expert consensus document: Cholangiocarcinoma: current knowledge and future perspectives consensus statement from the European Network for the Study of Cholangiocarcinoma (ENS-CCA). Nat Rev Gastroenterol Hepatol. 2016;13:261-80.

4. Valle J, Wasan H, Palmer DH, Cunningham D, Anthoney A, Maraveyas A, et al. Cisplatin plus gemcitabine versus gemcitabine for biliary tract cancer. N Engl J Med. 2010;362:1273-81.

5. Ostuni R, Kratochvill F, Murray PJ, Natoli G. Macrophages and cancer: from mechanisms to therapeutic implications. Trends Immunol. 2015:36:229-39.

6. Qian BZ, Pollard JW. Macrophage diversity enhances tumor progression and metastasis. Cell. 2010;141:39-51.

7. Yeung OW, Lo CM, Ling CC, Qi X, Geng W, Li CX, et al. Alternatively activated (M2) macrophages promote tumour growth and invasiveness in hepatocellular carcinoma. J Hepatol. 2015;62:607-16.

8. Hsu DS, Wang HJ, Tai SK, Chou CH, Hsieh CH, Chiu PH, et al. Acetylation of snail modulates the cytokinome of cancer cells to enhance the recruitment of macrophages. Cancer Cell. 2014;26:534-48.

9. Sirica AE, Gores GJ. Desmoplastic stroma and cholangiocarcinoma: clinical implications and therapeutic targeting. Hepatology. 2014:59:2397-402.

10. Gentilini A, Pastore M, Marra F, Raggi C. The role of stroma in cholangiocarcinoma: the intriguing interplay between fibroblastic component, immune cell subsets and tumor epithelium. Int J Mol Sci. 2018;19(10):2885.

11. Kitano Y, Okabe H, Yamashita Yl, Nakagawa S, Saito Y, Umezaki N, et al. Tumour-infiltrating inflammatory and immune cells in patients with extrahepatic cholangiocarcinoma. Br J Cancer. 2018;118:171-80.

12. Turley SJ, Cremasco V, Astarita JL. Immunological hallmarks of stromal cells in the tumour microenvironment. Nat Rev Immunol. 2015;15:669-82.

13. Ijichi H, Chytil A, Gorska AE, Aakre ME, Bierie B, Tada M, et al. Inhibiting Cxcr2 disrupts tumor-stromal interactions and improves survival in a mouse model of pancreatic ductal adenocarcinoma. J Clin Invest. 2011;121:4106-17.

14. Thiery JP, Acloque H, Huang RY, Nieto MA. Epithelial-mesenchymal transitions in development and disease. Cell. 2009;139:871-90. 
15. Qian Y, Yao W, Yang T, Yang Y, Liu Y, Shen Q, et al. aPKC-iota/P-Sp1/Snail signaling induces epithelial-mesenchymal transition and immunosuppression in cholangiocarcinoma. Hepatology. 2017;66:1165-82.

16. Yang T, Li B, Qi S, Liu Y, Gai Y, Ye P, et al. Co-delivery of doxorubicin and Bmi1 siRNA by folate receptor targeted liposomes exhibits enhanced anti-tumor effects in vitro and in vivo. Theranostics. 2014;4:1096-111.

17. Rahman A, Husain SR, Siddiqui J, Verma M, Agresti M, Center M, et al. Liposome-mediated modulation of multidrug resistance in human $\mathrm{HL}-60$ leukemia cells. J Natl Cancer Inst. 1992;84:1909-15.

18. Zhao X, Li F, Li Y, Wang H, Ren H, Chen J, et al. Co-delivery of HIF1alpha siRNA and gemcitabine via biocompatible lipid-polymer hybrid nanoparticles for effective treatment of pancreatic cancer. Biomaterials. 2015:46:13-25.

19. Murray NR, Fields AP. Atypical protein kinase C iota protects human leukemia cells against drug-induced apoptosis. J Biol Chem. 1997;272:27521-4.

20. Paul A, Gunewardena S, Stecklein SR, Saha B, Parelkar N, Danley M, et al. PKClambda/iota signaling promotes triple-negative breast cancer growth and metastasis. Cell Death Differ. 2014;21:1469-81.

21. Kusne Y, Carrera-Silva EA, Perry AS, Rushing EJ, Mandell EK, Dietrich JD, et al. Targeting aPKC disables oncogenic signaling by both the EGFR and the proinflammatory cytokine TNFalpha in glioblastoma. Sci Signal. 2014;7:ra75.

22. Sanz L, Diaz-Meco MT, Nakano H, Moscat J. The atypical PKC-interacting protein p62 channels NF-kappaB activation by the IL-1-TRAF6 pathway. EMBO J. 2000;19:1576-86.

23. Zhang Q, Lenardo MJ, Baltimore D. 30 Years of NF-kappaB: A Blossoming of Relevance to Human Pathobiology. Cell. 2017;168:37-57.

24. Karin M. Nuclear factor-kappaB in cancer development and progression. Nature. 2006;441:431-6.

25. Nakanishi C, Toi M. Nuclear factor-kappaB inhibitors as sensitizers to anticancer drugs. Nat Rev Cancer. 2005;5:297-309.

26. Song $R$, Song $H$, Liang $Y$, Yin D, Zhang H, Zheng T, et al. Reciprocal activation between ATPase inhibitory factor 1 and NF-kappaB drives hepatocellular carcinoma angiogenesis and metastasis. Hepatology. 2014;60:1659-73

27. Huber MA, Azoitei N, Baumann B, Grunert S, Sommer A, Pehamberger $H$, et al. NF-kappaB is essential for epithelial-mesenchymal transition and metastasis in a model of breast cancer progression. J Clin Invest. 2004;114:569-81.

28. Hirano Y, Yoshinaga S, Ogura K, Yokochi M, Noda Y, Sumimoto H, et al. Solution structure of atypical protein kinase C PB1 domain and its mode of interaction with ZIP/p62 and MEK5. J Biol Chem. 2004;279:31883-90.

29. Wilson MI, Gill DJ, Perisic O, Quinn MT, Williams RL. PB1 domain-mediated heterodimerization in NADPH oxidase and signaling complexes of atypical protein kinase C with Par6 and p62. Mol Cell. 2003;12:39-50.

30. Lamark T, Perander M, Outzen H, Kristiansen $K$, Overvatn A, Michaelsen E, et al. Interaction codes within the family of mammalian Phox and Bem1p domain-containing proteins. J Biol Chem. 2003;278:34568-81.

31. Wooten MW, Geetha T, Seibenhener ML, Babu JR, Diaz-Meco MT, Moscat J. The p62 scaffold regulates nerve growth factor-induced NF-kappaB activation by influencing TRAF6 polyubiquitination. J Biol Chem. 2005;280:35625-9.

32. Massague J. TGFbeta in Cancer. Cell. 2008;134:215-30.

33. Yang Y, Liu Y, He JC, Wang JM, Schemmer $P$, Ma CQ, et al. 14-3-3zeta and aPKC-iota synergistically facilitate epithelial-mesenchymal transition of cholangiocarcinoma via GSK-3beta/Snail signaling pathway. Oncotarget. 2016;7:55191-210.

34. Genin P, Algarte M, Roof P, Lin R, Hiscott J. Regulation of RANTES chemokine gene expression requires cooperativity between NFkappa B and IFN-regulatory factor transcription factors. J Immunol. 2000;164:5352-61.

35. Azenshtein E, Luboshits G, Shina S, Neumark E, Shahbazian D, Weil M, et al. The CC chemokine RANTES in breast carcinoma progression: regulation of expression and potential mechanisms of promalignant activity. Cancer Res. 2002;62:1093-102

36. Frankenberger C, Rabe D, Bainer R, Sankarasharma D, Chada K, Krausz T, et al. Metastasis Suppressors Regulate the Tumor Microenvironment by Blocking Recruitment of Prometastatic Tumor-Associated Macrophages. Cancer Res. 2015;75:4063-73.

37. Velasco-Velazquez M, Jiao X, De La Fuente M, Pestell TG, Ertel A, Lisanti MP, et al. CCR5 antagonist blocks metastasis of basal breast cancer cells Cancer Res. 2012;72:3839-50.
38. Nakasone ES, Askautrud HA, Kees T, Park JH, Plaks V, Ewald AJ, et al. Imaging tumor-stroma interactions during chemotherapy reveals contributions of the microenvironment to resistance. Cancer Cell. 2012;21:488-503.

39. Desai S, Pillai P, Win-Piazza H, Acevedo-Duncan M. PKC-iota promotes glioblastoma cell survival by phosphorylating and inhibiting BAD through a phosphatidylinositol 3-kinase pathway. Biochim Biophys Acta. 2011;1813:1190-7.

40. Wooten MW, Seibenhener ML, Zhou G, Vandenplas ML, Tan TH. Overexpression of atypical PKC in PC12 cells enhances NGF-responsiveness and survival through an NF-kappaB dependent pathway. Cell Death Differ. 1999;6:753-64.

41. Liu B, Yan S, Jia Y, Ma J, Wu S, Xu Y, et al. TLR2 promotes human intrahepatic cholangiocarcinoma cell migration and invasion by modulating NF-kappaB pathway-mediated inflammatory responses. FEBS J. 2016;283:3839-50.

42. Seubwai W, Wongkham C, Puapairoj A, Khuntikeo N, Pugkhem A, Hahnvajanawong C, et al. Aberrant expression of NF-kappaB in liver fluke associated cholangiocarcinoma: implications for targeted therapy. PLoS One. 2014;9:e106056.

43. Smith MP, Sanchez-Laorden B, O'Brien K, Brunton H, Ferguson J, Young H, et al. The immune microenvironment confers resistance to MAPK pathway inhibitors through macrophage-derived TNFalpha. Cancer Discov. 2014;4:1214-29.

44. Weizman N, Krelin Y, Shabtay-Orbach A, Amit M, Binenbaum Y, Wong $\mathrm{RJ}$, et al. Macrophages mediate gemcitabine resistance of pancreatic adenocarcinoma by upregulating cytidine deaminase. Oncogene. 2014;33:3812-9.

45. Erdogan E, Lamark T, Stallings-Mann M, Lee J, Pellecchia M, Thompson EA, et al. Aurothiomalate inhibits transformed growth by targeting the PB1 domain of protein kinase Ciota. J Biol Chem. 2006;281:28450-9.

46. Wyckoff J, Wang W, Lin EY, Wang Y, Pixley F, Stanley ER, et al. A paracrine loop between tumor cells and macrophages is required for tumor cell migration in mammary tumors. Cancer Res. 2004;64:7022-9.

47. Kumar V, Donthireddy L, Marvel D, Condamine T, Wang F, Lavilla-Alonso $S$, et al. Cancer-Associated Fibroblasts Neutralize the Anti-tumor Effect of CSF1 Receptor Blockade by Inducing PMN-MDSC Infiltration of Tumors. Cancer Cell. 2017:32:654-68 e5.

48. Su S, Liu Q, Chen J, Chen J, Chen F, He C, et al. A positive feedback loop between mesenchymal-like cancer cells and macrophages is essential to breast cancer metastasis. Cancer Cell. 2014;25:605-20.

49. Acharyya S, Oskarsson T, Vanharanta S, Malladi S, Kim J, Morris PG, et al. A CXCL1 paracrine network links cancer chemoresistance and metastasis. Cell. 2012;150:165-78.

\section{Publisher's Note}

Springer Nature remains neutral with regard to jurisdictional claims in published maps and institutional affiliations. 